\title{
Cloud-scale ISM Structure and Star Formation in M51
}

\author{
Adam K. Leroy ${ }^{1}$ (D) Eva Schinnerer $^{2}$ (D), Annie Hughes ${ }^{3,4}$ (D) J. M. Diederik Kruijssen ${ }^{5,2}$, Sharon Meidt ${ }^{2}$ (D), Andreas Schruba ${ }^{6}$, \\ Jiayi Sun $^{1}$ (D), Frank Bigiel ${ }^{7}$ (D), Gonzalo Aniano ${ }^{8}$, Guillermo A. Blanc ${ }^{9,10,11}$, Alberto Bolatto ${ }^{12}$ (D), Mélanie Chevance ${ }^{5}$, \\ Dario Colombo $^{13}$, Molly Gallagher ${ }^{1}$ (D), Santiago Garcia-Burillo ${ }^{14}$ (D) , Carsten Kramer ${ }^{15}$, Miguel Querejeta ${ }^{16}$, Jerome Pety ${ }^{17,18}$ (iD), \\ Todd A. Thompson ${ }^{1,19}$, and Antonio Usero ${ }^{14}$ (iD \\ ${ }^{1}$ Department of Astronomy, The Ohio State University, 140 West 18th Avenue, Columbus, OH 43210, USA \\ ${ }^{2}$ Max-Planck-Institut für Astronomie, Königstuhl 17, D-69117, Heidelberg, Germany \\ ${ }^{3}$ CNRS, IRAP, 9 av. du Colonel Roche, BP 44346, F-31028 Toulouse cedex 4, France \\ ${ }^{4}$ Université de Toulouse, UPS-OMP, IRAP, F-31028 Toulouse cedex 4, France \\ 5 Astronomisches Rechen-Institut, Zentrum für Astronomie der Universität Heidelberg, Mönchhofstraße 12-14, D-69120 Heidelberg, Germany \\ ${ }^{6}$ Max-Planck-Institut für extraterrestrische Physik, Giessenbachstraße 1, D-85748 Garching, Germany \\ ${ }^{7}$ Institute für theoretische Astrophysik, Zentrum für Astronomie der Universität Heidelberg, Albert-Ueberle Str. 2, D-69120 Heidelberg, Germany \\ 8 Princeton University Observatory, Peyton Hall, Princeton, NJ 08544-1001, USA \\ ${ }^{9}$ Departamento de Astronomía, Universidad de Chile, Casilla 36-D, Santiago, Chile \\ ${ }^{10}$ Centro de Astrofísica y Tecnologías Afines (CATA), Camino del Observatorio 1515, Las Condes, Santiago, Chile \\ ${ }^{11}$ Visiting Astronomer, Observatories of the Carnegie Institution for Science, 813 Santa Barbara Street, Pasadena, CA 91101, USA \\ ${ }^{12}$ Department of Astronomy, Laboratory for Millimeter-wave Astronomy, and Joint Space Institute, University of Maryland, College Park, MD 20742, USA \\ ${ }^{13}$ Max-Planck-Institut für Radioastronomie, Auf dem Hügel 69, D-53121 Bonn, Germany \\ ${ }^{14}$ Observatorio Astronómico Nacional (IGN), C/Alfonso XII, 3, E-28014 Madrid, Spain \\ ${ }^{15}$ Instituto Radioastronomía Milimétrica (IRAM), Av. Divina Pastora 7, Nucleo Central, E-18012 Granada, Spain \\ ${ }^{16}$ European Southern Observatory, Karl-Schwarzschild-Straße 2, D-85748 Garching, Germany \\ ${ }^{17}$ Institut de Radioastronomie Millimètrique (IRAM), 300 Rue de la Piscine, F-38406 Saint Martin d'Hères, France \\ ${ }^{18}$ Observatoire de Paris, 61 Avenue de l'Observatoire, F-75014 Paris, France \\ ${ }^{19}$ Center for Cosmology \& Astro-Particle Physics, The Ohio State University, Columbus, OH 43210, USA \\ Received 2017 February 8; revised 2017 June 12; accepted 2017 June 19; published 2017 August 31
}

\begin{abstract}
We compare the structure of molecular gas at $40 \mathrm{pc}$ resolution to the ability of gas to form stars across the disk of the spiral galaxy M51. We break the PAWS survey into $370 \mathrm{pc}$ and $1.1 \mathrm{kpc}$ resolution elements, and within each we estimate the molecular gas depletion time $\left(\tau_{\mathrm{Dep}}^{\mathrm{mol}}\right)$, the star-formation efficiency per free-fall time $\left(\epsilon_{\mathrm{ff}}\right)$, and the mass-weighted cloud-scale $(40 \mathrm{pc})$ properties of the molecular gas: surface density, $\Sigma$, line width, $\sigma$, and $b \equiv \Sigma / \sigma^{2} \propto \alpha_{\text {vir }}^{-1}$, a parameter that traces the boundedness of the gas. We show that the cloud-scale surface density appears to be a reasonable proxy for mean volume density. Applying this, we find a typical star-formation efficiency per free-fall time, $\epsilon_{\mathrm{ff}}\left(\left\langle\Sigma_{40 \mathrm{pc}}\right\rangle\right) \sim 0.3 \%-0.36 \%$, lower than adopted in many models and found for local clouds. Furthermore, the efficiency per free-fall time anti-correlates with both $\Sigma$ and $\sigma$, in some tension with turbulent star-formation models. The best predictor of the rate of star formation per unit gas mass in our analysis is $b \equiv \Sigma / \sigma^{2}$, tracing the strength of self-gravity, with $\tau_{\text {Dep }}^{\text {mol }} \propto b^{-0.9}$. The sense of the correlation is that gas with stronger self-gravity (higher $b$ ) forms stars at a higher rate (low $\tau_{\text {Dep }}^{\text {mol }}$ ). The different regions of the galaxy mostly

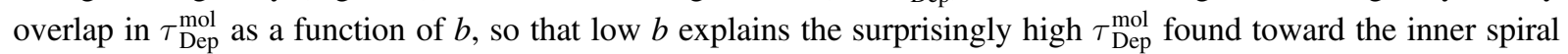
arms found by Meidt et al. (2013).
\end{abstract}

Key words: galaxies: individual (M51) - galaxies: ISM - galaxies: spiral - galaxies: star formation - ISM: general - ISM: structure

Supporting material: machine-readable table

\section{Introduction}

In the local universe, star formation occurs in molecular gas. The recent star-formation rate (SFR) correlates better with tracers of molecular gas than tracers of atomic gas (Bigiel et al. 2008; Leroy et al. 2008; Blanc et al. 2009; Schruba et al. 2011), even though atomic gas represents the dominant reservoir by mass of the interstellar medium (ISM) in galaxies at $z=0$ (e.g., Saintonge et al. 2011). But even within the molecular ISM of a galaxy, only a small fraction of the gas participates in star formation at any given time (e.g., Heiderman et al. 2010; Lada et al. 2010), and the properties of molecular gas vary among galaxies and among regions within galaxies (e.g., Hughes et al. 2013b; Leroy et al. 2016). The SFR per unit molecular gas mass should depend on these properties: e.g., the density, turbulence, and balance of potential and kinetic energy. As a result, we expect star formation to proceed at different specific (per unit gas mass) rates in different environments.

Observations indeed indicate that the SFR per unit molecular gas mass does vary across the local galaxy population (Young et al. 1996). High stellar mass, early-type galaxies show comparatively low SFRs per unit $\mathrm{H}_{2}$ mass (Saintonge et al. 2011; Leroy et al. 2013; Davis et al. 2014). Starbursts, especially galaxy-wide bursts induced by major galaxy mergers, have a high SFR per unit $\mathrm{H}_{2}$ mass (e.g., Kennicutt 1998; Gao \& Solomon 2004). So do some galaxy centers (e.g., Leroy et al. 2013, 2015). Low stellar mass, low metallicity, late-type galaxies exhibit a high SFR per unit CO emission (e.g., Young et al. 1996; Schruba et al. 2012, 2017; Leroy et al. 2013). Although the translation of $\mathrm{CO}$ emission into $\mathrm{H}_{2}$ mass remains uncertain in these systems (Bolatto et al. 2013a), 
several works argue that the SFR per $\mathrm{H}_{2}$ mass is indeed higher in these systems (e.g., Gardan et al. 2007; Bothwell et al. 2014; Hunt et al. 2015). Within galaxies, dynamical effects can both enhance (Koda et al. 2009; Suwannajak et al. 2014) and suppress (Meidt et al. 2013) the SFR-per-H $\mathrm{H}_{2}$. As our ability to observe the molecular ISM across diverse environments improves, the list of observed variations in the SFR per unit $\mathrm{H}_{2}$ mass continues to grow.

Though driven by large-scale environmental factors, the observed SFR-per- $\mathrm{H}_{2}$ variations must have their immediate origins in the properties of the clouds that host star formation. That is, in an environment with a high SFR per unit gas mass, we expect the configuration and small-scale physical properties of the molecular ISM to be more conducive to star formation.

Recent theoretical work exploring variations in SFR-per- $\mathrm{H}_{2}$ has focused on the properties of turbulent molecular clouds. In such models, the mean density, gravitational boundedness, and Mach number of a cloud determine its normalized rate of star formation (e.g., Padoan \& Nordlund 2002, 2011; Krumholz \& McKee 2005; Hennebelle \& Chabrier 2011; Federrath \& Klessen 2012, 2013). These properties set the density structure of the cloud and the balance between kinetic and potential energy, determining the fraction of the gas in a directly starforming, self-gravitating component. In such models, the gravitational free-fall time, $\tau_{\mathrm{ff}} \propto \rho^{-0.5}$, often emerges as the characteristic timescale for star formation at many scales (e.g., Krumholz \& McKee 2005), albeit with a low efficiency per $\tau_{\mathrm{ff}}$ (see also McKee \& Ostriker 2007).

Observations and theory suggest that the turbulent motions in molecular clouds are driven at about at the scale of an individual cloud $(d \approx 30-100$ pc, e.g., Brunt 2003; Mac Low \& Klessen 2004; Brunt et al. 2009) making this the relevant scale for many of the models referenced above. Current millimeter-wave telescopes can observe the structure of molecular gas at these scales across large areas of galaxies. This allows the prospect to measure how the cloud-scale structure of the cold ISM relates to the ability of gas to form stars in different galactic environments.

In this paper, we carry out such a study targeting M51. Our key data set is the PdBI Arcsecond Whirlpool Survey ${ }^{20}$ (PAWS, Schinnerer et al. 2013). PAWS mapped CO (1-0) emission from the inner $9 \times 6 \mathrm{kpc}$ of M51 at $40 \mathrm{pc}$ resolution (adopting a distance of 7.6 Mpc; Feldmeier et al. 1997; Ciardullo et al. 2002). From PAWS, we know the structure of the turbulent ISM at the scale of an individual giant molecular cloud (GMC, see Hughes et al. 2013a, 2013b; Colombo et al. 2014a). Combining this information with infrared maps from Herschel and Spitzer (Kennicutt et al. 2003; Mentuch Cooper et al. 2012), we measure how the cloud-scale structure of the ISM relates to M51's ability to form stars.

This analysis builds on studies by Koda et al. (2009), Hughes et al. (2013a, 2013b), and Colombo et al. (2014a), which showed that the cloud-scale ISM structure in M51 depends on environment. We also follow Meidt et al. (2013), Liu et al. (2011), Momose et al. (2013), and Shetty et al. (2013), who compare gas and star formation in M51 and came to apparently contradictory conclusions regarding whether star formation proceeds more quickly or more slowly in the highest density regions. In particular, we follow Meidt et al. (2013)

\footnotetext{
${ }^{20}$ This work is based on observations carried out with the IRAM NOEMA Interferometer and the IRAM $30 \mathrm{~m}$ telescope. IRAM is supported by INSU/ CNRS (France), MPG (Germany), and IGN (Spain).
}

who also compared PAWS to infrared (IR) data, focusing on the impact of dynamics on the ability of gas to form stars.

We use the methodology described by Leroy et al. (2016). In this approach, we calculate the molecular gas depletion time, $\tau_{\text {Dep }}^{\text {mol }} \equiv M_{\text {mol }} /$ SFR, averaged over a moderate-sized area, $\theta=370-1100 \mathrm{pc}$, and compare this to the mass-weighted $40 \mathrm{pc}$ surface density, line width, and self-gravity (virial parameter) within the larger beam. This approach captures both ensemble averages and local physical conditions. We expect

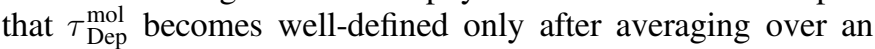
ensemble of star-forming regions in different evolutionary states (e.g., see Schruba et al. 2010; Kruijssen \& Longmore 2014). Meanwhile the beam-by-beam $40 \mathrm{pc}$ structural measurements from PAWS allow us to test expectations from turbulent theories. By taking the mass-weighted average within each larger beam, we preserve the small-scale structural information in the PAWS map.

\section{Methods}

We wish to measure how small-scale ISM structure relates to the ability of gas to form stars in M51. To do this, we require region-by-region estimates of the recent SFR, the molecular gas reservoir, and the structure of molecular gas on the scale of an individual cloud. Using these, we correlate the cloud-scale structure of the molecular gas with the SFR per unit gas mass, expressed as a gas depletion time, $\tau_{\text {Dep }}^{\text {mol }}$.

We estimate these quantities and conduct a correlation analysis at $30^{\prime \prime}$ and $10^{\prime \prime}$ resolution. These correspond to linear resolutions of $\sim 1100 \mathrm{pc}$ and $\sim 370 \mathrm{pc}$ at our adopted distance of 7.6 Mpc to M51 (Feldmeier et al. 1997; Ciardullo et al. 2002). At $30^{\prime \prime}$ resolution, we are able to include more IR bands in our SFR estimate. At $10^{\prime \prime}$ resolution, we are better able to resolve the dynamical features that drive the differences within the M51 cloud population (Koda et al. 2009; Meidt et al. 2013, 2015). At resolutions finer than 10", we cannot include infrared (IR) emission, our main SFR indicator (see the Appendix).

The choice of a few hundred parsecs to a kiloparsec scale also ensures that within a resolution element we average over many individual star-forming regions. This allows us to avoid most effects related to the time evolution of individual regions (see Kawamura et al. 2009; Schruba et al. 2010; Kruijssen \& Longmore 2014), and thus to better access the time-averaged behavior of the ISM. The evolutionary effects revealed at high resolution are explored in E. Schinnerer et al. (2017, in preparation) and M. Chevance et al. (2017, in preparation).

In practice, we record the properties of M51 at each point in a hexagonally packed, half-beam-spaced grid (see Leroy et al. 2013). Figure 1 shows the individual sampling points for these two grids, overlaid on the Herschel $70 \mu \mathrm{m}$ map (Mentuch Cooper et al. 2012).

\subsection{Data}

Schinnerer et al. (2013) and Pety et al. (2013) present PAWS, which mapped CO (1-0) emission from the central region of M51 at 1 "! $16 \times 0$ "! $97 \sim 1$ " $06 \sim 40 \mathrm{pc}$ resolution with $\sim 5 \mathrm{~km} \mathrm{~s}^{-1}$ velocity resolution. PAWS includes short and zero-spacing information. Schinnerer et al. (2013) also summarize the multiwavelength data available for M51, with references (see their Table 2).

We also use broad-band maps of IR emission from Herschel and Spitzer. These were obtained as part of the Spitzer Infrared 


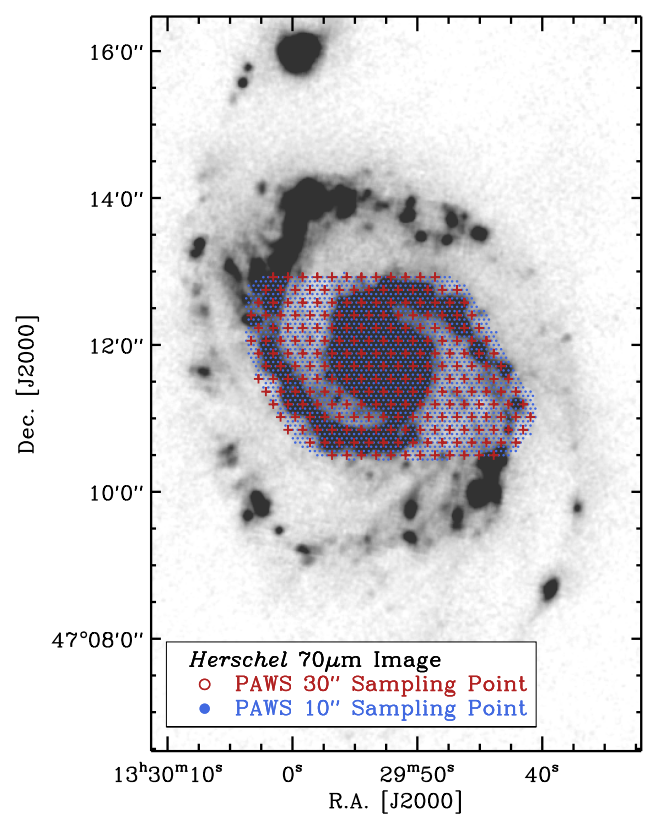

Figure 1. Sampling points used in this paper, overlaid on the Herschel $70 \mu \mathrm{m}$ image of M51 (Mentuch Cooper et al. 2012). The points are hexagonally packed and spaced by $15^{\prime \prime}$ (red) and $5^{\prime \prime}$ (blue), corresponding to half-beam spacing for our two working resolutions. The area studied is set by the PAWS field (Schinnerer et al. 2013). The coarser resolution $30^{\prime \prime}$ data allow the inclusion of all the IR bands.

Nearby Galaxy Survey (Kennicutt et al. 2003) and the Herschel Very Nearby Galaxies Survey (Mentuch Cooper et al. 2012).

\subsection{Measurements}

Integrated CO intensity: At $30^{\prime \prime}$ resolution, we use the PAWS single dish map (Pety et al. 2013) to measure the integrated CO intensity. At $10^{\prime \prime}$, we convolve the combined interferometer and single dish cube to a coarser $10^{\prime \prime}$ resolution and measure the integrated intensity from this degraded map. As discussed by Pety et al. (2013), the deconvolution of the hybrid 30m+PdBI map recovers $99 \%$ flux of the galaxy observed with the IRAM $30 \mathrm{~m}$.

To collapse the $30^{\prime \prime}$ and $10^{\prime \prime}$ cubes to integrated intensity measurements, we sum over a broad velocity window from -70 to $+70 \mathrm{~km} \mathrm{~s}^{-1}$ about the local mean velocity. The signalto-noise in $\mathrm{CO}(1-0)$ is very high, so some empty bandwidth is not a concern. We estimate the associated uncertainty by measuring the rms noise of the convolved line cube from the signal-free region. Then the statistical uncertainty in the integrated intensity is the sum in quadrature of the per-channel intensity noise across all channels in the velocity integration window multiplied by the velocity width of a channel.

Total infrared (TIR) surface brightness: We convolve the IR data to have Gaussian beams using the kernels of Aniano et al. (2011). Then, using the formulae of Galametz et al. (2013), we combine Spitzer $24 \mu \mathrm{m}$ and $70 \mu \mathrm{m}$ intensities with Herschel $160 \mu \mathrm{m}$ and $250 \mu \mathrm{m}$ intensities to estimate a TIR luminosity surface brightness, $\Sigma_{\mathrm{TIR}}$, for each resolution element. This is our basic measure of star-formation activity throughout this paper.

At $10^{\prime \prime}$ resolution, we can only use the Herschel $70 \mu \mathrm{m}$ data. We calculate the coefficient to translate $I_{70}$ to $\Sigma_{\text {TIR }}$ by comparing the two quantities at $30^{\prime \prime}$ resolution, where we know $\Sigma_{\mathrm{TIR}}$ from the four-band calculation following Galametz et al. (2013). In the PAWS field, the ratio $\Sigma_{\mathrm{TIR}} / I_{70}$ varies modestly as a function of radius, presumably reflecting a radial change in dust temperature. We find

$$
\begin{gathered}
\frac{\Sigma_{\mathrm{TIR}}}{I_{70}}=10^{6}\left\{\begin{array}{l}
f\left(r_{\mathrm{gal}}\right) \text { if } r_{\mathrm{gal}}<2.5 \mathrm{kpc} \\
2.96 \text { if } r_{\mathrm{gal}}>2.5 \mathrm{kpc}
\end{array}\right. \\
\text { where } f(r)=1.93+0.01 r+0.28 r^{2}-0.048 r^{3} .
\end{gathered}
$$

Here $r_{\text {gal }}$ refers to the deprojected galactocentric radius and $f(r)$ is a polynomial fit to the ratio $\Sigma_{\mathrm{TIR}} / I_{70}$ as a function of $r$ inside $r_{\mathrm{gal}}=2.5 \mathrm{kpc} . \Sigma_{\mathrm{TIR}}$ has units of $L_{\odot} \mathrm{kpc}^{-2}, I_{70}$ has units of $\mathrm{MJy} \mathrm{sr}^{-1}$, and $r_{\mathrm{gal}}$ has units of kpc. Outside $r_{\mathrm{gal}} \sim 2.5 \mathrm{kpc}$, the ratio appears flat. The Appendix compares SFRs derived from $I_{70}$ using this approach to those using $\Sigma_{\mathrm{TIR}}$ at $\theta=30^{\prime \prime}$. The two show a median ratio of 1 , less than $10 \%$ scatter and no clear systematics across the PAWS field.

At $30^{\prime \prime}$, we measure the rms scatter in $\Sigma_{\mathrm{TIR}}$ from the low intensity regions of the map to be $\sim 2.5 \times 10^{6} L_{\odot} \mathrm{kpc}^{-2}$. At $10^{\prime \prime}$, using only the $70 \mu \mathrm{m}$ data, the rms scatter is higher, $\sim 8.5 \times 10^{6} L_{\odot} \mathrm{kpc}^{-2}$.

Cloud-scale properties: We measure the intensity-weighted cloud-scale properties of the gas in each beam following Leroy et al. (2016). In brief, we begin with the native $40 \mathrm{pc}$ resolution PAWS cube. We recenter each spectrum about the local mean velocity. Next, we weight each spectrum by the integrated intensity along the line of sight and convolve from $1^{\prime \prime} \approx 40 \mathrm{pc}$ resolution (our "measurement scale") to $10^{\prime \prime} \approx 370 \mathrm{pc}$ or $30^{\prime \prime} \approx 1.1 \mathrm{kpc}$ (our "averaging scales"). From these intensity-weighted, stacked spectra, we measure the integrated intensity and line width of the gas. This cross-scale weighted averaging also resembles that by Ossenkopf \& Mac Low (2002).

Because of the intensity ( $\sim$ mass for fixed $\alpha_{\mathrm{CO}}$ ) weighting, this approach captures the high-resolution structure of the emission within each larger averaging beam. Leroy et al. (2016) demonstrated that the results match those from massweighted averages of cloud catalogs well, but with far fewer assumptions. We write the resulting measurements as, e.g., $\left\langle\Sigma_{40} \mathrm{pc}\right\rangle$. This is read as "the mass-weighted average $40 \mathrm{pc}$ surface density within a larger beam. ${ }^{21}$ " We focus on three such measurements.

1. The cloud-scale molecular gas surface density, $\left\langle\Sigma_{40 \mathrm{pc}}\right\rangle$. This is a linear translation of the integrated intensity, $\left\langle\Sigma_{40 \mathrm{pc}}\right\rangle=\alpha_{\mathrm{CO}}\left\langle I_{40 \mathrm{pc}}\right\rangle$, where $\alpha_{\mathrm{CO}}$ is our adopted $\mathrm{CO}$-to- $\mathrm{H}_{2}$ conversion factor. If the line-of-sight length of the gas distribution, $h$, is known or assumed, then $\left\langle\Sigma_{40 \mathrm{pc}}\right\rangle$ can be used to estimate the volume density of the gas on $40 \mathrm{pc} \times h$ scales, $\rho\left(\left\langle\Sigma_{40 \mathrm{pc}}\right\rangle\right)$. From this, one can estimate the gravitational free-fall time, $\left\langle\tau_{\mathrm{ff}, 40 \mathrm{pc}}\right\rangle$. We show in Section 3.2.2 that for published Milky Way and M51 cloud catalogs, $\Sigma_{\text {mol }}$ and $\rho$ do correlate well.

2. The rms line width of $C O,\left\langle\sigma_{40} \mathrm{pc}\right\rangle$, measured from the "equivalent width" and corrected for channelization and channel-to-channel correlation following Leroy et al. (2016). For a given temperature and when the line width is purely turbulent in nature, this corresponds to the turbulent Mach number, $\mathcal{M} .\left\langle\sigma_{40} \mathrm{pc}\right\rangle$ may also contain a

\footnotetext{
${ }^{21}$ More rigorously, following Leroy et al. (2016), we would also indicate the size of that larger beam (the "averaging scale") when quoting $\left\langle\Sigma_{40} \mathrm{pc}\right\rangle$. In this paper, the plots, discussion, and tables make it clear whether $\left\langle\Sigma_{40} \mathrm{pc}\right\rangle$ refers to an averaging scale of $370 \mathrm{pc}\left(\left\langle\Sigma_{40 \mathrm{pc}}\right\rangle_{370 \mathrm{pc}}\right.$ or $1.1 \mathrm{kpc}\left(\left\langle\Sigma_{40 \mathrm{pc}}\right\rangle_{1.1 \mathrm{kpc}}\right.$.
} 
contribution from bulk motions unresolved at the $40 \mathrm{pc}$ resolution of PAWS (Meidt et al. 2013; Colombo et al. 2014b, and S. Meidt et al., submitted). Thermal contributions to the line width are expected to be small.

3. The dynamical state of the gas, as traced by the ratio $\left\langle b_{40 \mathrm{pc}}\right\rangle$ with $b \equiv \Sigma_{\mathrm{mol}} / \sigma^{2}$. This "boundedness parameter" also relies on an adopted $\mathrm{CO}$-to- $\mathrm{H}_{2}$ conversion factor. Within a length scale, $b$ is proportional to $\mathrm{UE} / \mathrm{KE}$, the ratio of potential energy (UE) to kinetic energy (KE). This is the inverse of the virial parameter, $b^{-1} \propto$ $\alpha_{\text {vir }} \approx 2 \mathrm{KE} / \mathrm{UE}$. When $b$ is high, the gas should be more gravitationally bound.

Also, within a length scale $\left\langle b_{40 \mathrm{pc}}\right\rangle \propto \tau_{\text {cross }}^{2} / \tau_{\mathrm{ff}}^{2}$, where $\tau_{\mathrm{ff}} \propto 1 / \sqrt{M / R^{3}}$ is the free-fall time and $\tau_{\text {cross }} \sim$ $R / \sigma$ is the crossing time for the measured velocity dispersion. This ratio has been highlighted by Padoan et al. (2012) as a key driver for the star-formation efficiency per free-fall time.

Note that in this paper we focus on $b \equiv \Sigma_{\text {mol }} / \sigma^{2}$. This differs from the $B \equiv I_{\mathrm{CO}} / \sigma^{2}$ discussed in Leroy et al. (2016) by a factor of $\alpha_{\mathrm{CO}}$, so that $b=\alpha_{\mathrm{CO}} B$. While $B$ has the advantage of being directly computed from observable quantities, $b \propto \alpha_{\text {vir }}^{-1}$ is more closely linked to the physical state of the gas.

We estimate uncertainties in $\left\langle\Sigma_{40 \mathrm{pc}}\right\rangle,\left\langle\sigma_{40 \mathrm{pc}}\right\rangle$, and $\left\langle b_{40 \mathrm{pc}}\right\rangle$ using a Monte Carlo approach. We measure the noise in the stacked, shuffled intensity-weighted spectra from the signalfree region. Then we realize 100 versions of each spectrum, adding random noise to the real spectrum. For each case, we remeasure $\left\langle\Sigma_{40 \mathrm{pc}}\right\rangle,\left\langle\sigma_{40 \mathrm{pc}}\right\rangle$, and $\left\langle b_{40 \mathrm{pc}}\right\rangle$. We compare these to our measurements without added noise, which we take to be the true values for purpose of this exercise. The rms offset between the simulated noisy data and the true value yields our estimate of the noise. This approach is ad hoc but yields realistic statistical uncertainties and captures the covariance among the uncertainties on $\left\langle\Sigma_{40 \mathrm{pc}}\right\rangle,\left\langle\sigma_{40 \mathrm{pc}}\right\rangle$, and $\left\langle b_{40 \mathrm{pc}}\right\rangle$.

\subsection{Conversion to Physical Parameters}

We report our results in terms of simple transformations of observable quantities into physical parameters.

Galactocentric coordinates: Following Schinnerer et al. (2013), we assume an inclination $i=22^{\circ}$ (Colombo et al. 2014b) and a position angle P.A. $=172^{\circ}$ (Colombo et al. 2014b) with the galaxy center at $\alpha_{2000}=13^{\mathrm{h}} 29^{\mathrm{m}} 52.7^{\mathrm{s}}, \delta_{2000}=$ $+47^{\circ} 11^{\prime} 43^{\prime \prime}$ (Hagiwara 2007). We adopt the $7.6 \mathrm{Mpc}$ distance of Feldmeier et al. (1997) and Ciardullo et al. (2002).

$\mathrm{CO}(1-0)$-to- $\mathrm{H}_{2}$ : We estimate $\mathrm{H}_{2}$ mass from $\mathrm{CO}(1-0)$ emission using a CO-to- $\mathrm{H}_{2}$ conversion factor $\alpha_{\mathrm{CO}}=4.35$ $M_{\odot} \mathrm{pc}^{-2}\left(\mathrm{~K} \mathrm{~km} \mathrm{~s}^{-1}\right)^{-1}$, which includes helium. This is a standard value for the Galaxy (Bolatto et al. 2013b). In the Appendix, we show that a dust-based approach following Sandstrom et al. (2013) and Leroy et al. (2011) suggests approximately this value. Schinnerer et al. (2010) came to the same conclusion via a multi-line $\mathrm{CO}$ analysis of the spiral arms. B. Groves et al. (2017, in preparation) show that this value applies with only weak variations across the disk of M51a using several independent methods.

There have been other values suggested for M51, mostly lower than Galactic by a factor of $\approx 2$ based on dust observations (e.g., Nakai \& Kuno 1995; Wall et al. 2016, though see our Appendix). We discuss the impact of a lower conversion factor in the text.

$\Sigma_{\mathrm{TIR}}$ to $\Sigma_{\mathrm{SFR}}$ : When relevant, we recast the TIR surface brightness as an SFR surface density using the conversion of Murphy et al. (2011), which assumes a Kroupa (2001) initial mass function and reduces to

$$
\frac{\Sigma_{\mathrm{SFR}}}{M_{\odot} \mathrm{yr}^{-1} \mathrm{kpc}^{-2}} \approx 1.5 \times 10^{-10} \frac{\Sigma_{\mathrm{TIR}}}{L_{\odot} \mathrm{kpc}^{-2}}
$$

A large body of work explores the subtleties of SFR estimation, often in M51 (e.g., Calzetti et al. 2005; Leroy et al. 2008, 2012; Blanc et al. 2009; Liu et al. 2011). Our focus in this paper is on new diagnostics of the molecular medium. Given the overwhelming extinction in the inner region of M51, we adopt the simple, widely accepted SFR diagnostic of TIR surface brightness. As a check, the Appendix shows the impact of several alternative SFR prescriptions on our inferred molecular gas depletion time at $30^{\prime \prime}$ resolution. These matter mainly to the overall normalization. By using the TIR emission, it is likely that we somewhat overestimate $\Sigma_{\mathrm{SFR}}$. One of our key findings is that $\epsilon_{\mathrm{ff}}\left(\left\langle\Sigma_{40 \mathrm{pc}}\right\rangle\right)$ is low (Section 3.2); this result would be even stronger if we used a tracer that yields lower $\Sigma_{\text {SFR }}$. The systematic trends appear weak and, when present, are opposite the sense needed to yield a fixed $\epsilon_{\mathrm{ff}}\left(\left\langle\Sigma_{40 \mathrm{pc}}\right\rangle\right)$. We intend to revisit this assumption in more detail in future work, ideally using an extinction-robust SFR tracer with high angular resolution to measure SFR on the scale of individual clouds.

\subsection{Dynamical Region}

Galactic dynamics relate to molecular gas structure and star formation in M51 (e.g., see Koda et al. 2009; Hughes et al. 2013a; Colombo et al. 2014a; Meidt et al. 2015; Schinnerer et al. 2017). With this in mind, we separate our correlation analysis by dynamical regions (Section 3.4). To do this, we use the dynamical region masks created by Colombo et al. (2014a). We use their simplified region definition, which breaks the PAWS field into "arm," "interarm," and "central" regions. For each $10^{\prime \prime}$ or $30^{\prime \prime}$ sampling point, we convolve the PAWS integrated $\mathrm{CO}$ intensity map multiplied by the mask for each separate region to the working resolution. Then we note the fraction of the flux in each beam coming from each dynamical region. When most of the $\mathrm{CO}$ flux in a beam comes from one dynamical environment, we associate the results for that beam with that environment.

Note that the three-region version of the Colombo et al. (2014a) mask may still group together physically distinct environments. We treat the upstream and downstream interarm regions together (e.g., see Meidt et al. 2015), and the "center" groups together the star-forming central molecular ring and the nucleus, which is more quiescent and potentially contaminated by the active galactic nucleus (AGN, e.g., see Querejeta et al. 2016). We report all of our measurements in Table 1.

\section{Results}

Figure 2 shows the scaling between TIR surface brightness, tracing $\Sigma_{\mathrm{SFR}}$, and $\mathrm{CO}$ intensity, tracing $\Sigma_{\text {mol }}$. The left panel shows all of M51 at $30^{\prime \prime} \approx 1.1 \mathrm{kpc}$ resolution. The right panel includes only data from the PAWS field, plotting the $10^{\prime \prime}$ resolution measurements in blue, the $30^{\prime \prime}$ measurements in red, 
Table 1

Cloud-scale Structure, IR, and CO in M51

\begin{tabular}{|c|c|c|c|c|c|c|c|c|c|c|c|}
\hline $\begin{array}{l}\text { R.A. } \\
\left({ }^{\circ}\right) \\
(1)\end{array}$ & $\begin{array}{l}\text { Decl. } \\
\left({ }^{\circ}\right) \\
(2)\end{array}$ & $\begin{array}{c}\text { Beam } \\
(") \\
(3)\end{array}$ & $\begin{array}{l}r_{\mathrm{gal}}{ }^{\mathrm{a}} \\
(\mathrm{kpc}) \\
(4)\end{array}$ & $\begin{array}{c}\Sigma_{\mathrm{mol}}{ }^{\mathrm{b}} \\
\left(M_{\odot} \mathrm{pc}^{-2}\right) \\
(5)\end{array}$ & $\begin{array}{c}\Sigma_{\mathrm{SFR}}{ }^{\mathrm{c}} \\
\left(\frac{M_{\odot \mathrm{yr}^{-1}}}{\mathrm{kpc}^{2}}\right) \\
(6)\end{array}$ & $\begin{array}{c}\left\langle\Sigma_{40 \mathrm{pc}}\right\rangle^{\mathrm{b}} \\
\left(M_{\odot} \mathrm{pc}^{-2}\right) \\
(7)\end{array}$ & $\begin{array}{c}\left\langle\sigma_{40 \mathrm{pc}}\right\rangle \\
\left(\mathrm{km} \mathrm{s}^{-1}\right) \\
(8)\end{array}$ & $\begin{array}{c}\left\langle b_{40 \mathrm{pc}}\right\rangle^{\mathrm{b}} \\
\left(\frac{M_{\odot} \mathrm{pc}^{-2}}{\left(\mathrm{~km} \mathrm{~s}^{-1}\right)^{2}}\right) \\
(9)\end{array}$ & $\begin{array}{l}f_{\text {arm }}{ }^{\mathrm{d}} \\
(\text { ) } \\
(10)\end{array}$ & $\begin{array}{l}f_{\mathrm{ia}}^{\mathrm{d}} \\
(\text { ) } \\
(11)\end{array}$ & $\begin{array}{l}f_{\text {ctr }}{ }^{\mathrm{d}} \\
(\text { ) } \\
(12)\end{array}$ \\
\hline 202.46718 & 47.19806 & 30 & 0.4 & 204.3 & 0.1909 & 445.5 & 10.5 & 4.03 & 0.02 & 0.02 & 0.97 \\
\hline 202.47209 & 47.19228 & 30 & 0.4 & 179.2 & 0.1688 & 353.2 & 9.9 & 3.57 & 0.03 & 0.01 & 0.96 \\
\hline 202.46718 & 47.19228 & 30 & 0.5 & 200.6 & 0.1698 & 412.8 & 11.1 & 3.37 & 0.07 & 0.00 & 0.92 \\
\hline 202.47453 & 47.19517 & 30 & 0.5 & 180.7 & 0.1775 & 365.5 & 10.2 & 3.52 & 0.02 & 0.00 & 0.97 \\
\hline 202.46964 & 47.18940 & 30 & 0.8 & 170.8 & 0.1339 & 367.6 & 10.4 & 3.40 & 0.15 & 0.02 & 0.83 \\
\hline 202.46964 & 47.20094 & 30 & 0.8 & 165.1 & 0.1493 & 394.9 & 9.6 & 4.33 & 0.10 & 0.04 & 0.86 \\
\hline 202.47699 & 47.19228 & 30 & 0.8 & 161.7 & 0.1482 & 383.6 & 10.3 & 3.62 & 0.02 & 0.02 & 0.96 \\
\hline
\end{tabular}

Notes. The following uncertainties apply: (a) uncertainty in the distance, $\sim 10 \%$, linearly affects $r_{\text {gal }}$, (b) for $\Sigma_{\text {mol }}$ a $\sim 10 \%$ gain uncertainty applies to both resolutions, the statistical noise is on average $2.25 M_{\odot} \mathrm{pc}^{-2}$ at $10^{\prime \prime}$ resolution and $0.5 \mathrm{M}_{\odot} \mathrm{pc}^{-2}$ at $30^{\prime \prime}$ resolution, (c) calibration uncertainties are of the order of 5\%-10\%, multiband TIR estimates from Galametz et al. (2013) are uncertain by $\sim 0.08$ dex, translation from $70 \mu \mathrm{m}$ to TIR scatters by an additional $\sim 0.05$ dex, and statistical noise is $\sim 2.5 \times 10^{6} L_{\odot} \mathrm{kpc}^{-2}\left(\approx 4 \times 10^{-4} M_{\odot} \mathrm{yr}^{-1} \mathrm{kpc}^{-2}\right)$ at $30^{\prime \prime}$ and $\sim 9.4 \times 10^{6} L_{\odot} \mathrm{kpc}^{-2}$ at $10^{\prime \prime}$ resolution $\left(\sim 1.3 \times 10^{-4} \mathrm{M}_{\odot} \mathrm{yr}^{-1} \mathrm{kpc}^{-2}\right)$, (d) from our Monte Carlo calculation, typical statistical uncertainties in $\left\langle\Sigma_{40 \mathrm{pc}}\right\rangle,\left\langle\sigma_{40 \mathrm{pc}}\right\rangle$, and $\left\langle b_{40} \mathrm{pc}\right\rangle$ are 1.5\%, 2\%, and 3\% at 30" resolution and 4\%, 5\%, and 6\% at $10^{\prime \prime}$ resolution. Covariance in uncertainty at both resolutions is about 0.7 between $\left\langle\Sigma_{40 \text { pc }}\right\rangle$ and $\left\langle\sigma_{40 \text { pc }}\right\rangle,-0.4$ between $\left\langle\Sigma_{40 \text { pc }}\right\rangle$ and $\left\langle b_{40 \text { pc }}\right\rangle$, and -0.9 between $\left\langle\sigma_{40 \text { pc }}\right\rangle$ and $\left\langle b_{40 \text { pc }}\right\rangle$. The $\sim 10 \%$ gain uncertainty also applies to $\left\langle\Sigma_{40 \mathrm{pc}}\right\rangle$ and $\left\langle b_{40} \mathrm{pc}\right\rangle$. These uncertainties do not account for translation to physical quantities. Selection criteria: at $30^{\prime \prime}$, we include all lines of sight where at least $50 \%$ of the beam lies in the PAWS field. At $10^{\prime \prime}$, we include all lines of sight where $95 \%$ of the beam lies in the PAWS field, $\Sigma_{\text {mol }}>5 M_{\odot}$ pc ${ }^{-2}$, and $\Sigma_{\mathrm{SFR}}>7.5 \times 10^{-3} M_{\odot} \mathrm{yr}^{-1} \mathrm{kpc}^{-2}$.

${ }^{a}$ Galactocentric radius for a thin disk and the orientation parameters quoted in Section 2.3.

${ }^{\mathrm{b}}$ Molecular mass linearly translated from CO surface brightness using $\alpha_{\mathrm{CO}}=4.35 M_{\odot} \mathrm{pc}^{-2}\left(\mathrm{~K} \mathrm{~km} \mathrm{~s}^{-1}\right)^{-1}$.

${ }^{\mathrm{c}}$ Here, the SFR is a linear transformation of the TIR emission. See Section 2.3.

${ }^{d}$ Fraction of the CO flux in the beam that arises from arm, interarm, or central regions as defined following Colombo et al. (2014a).

(This table is available in its entirety in machine-readable form.)
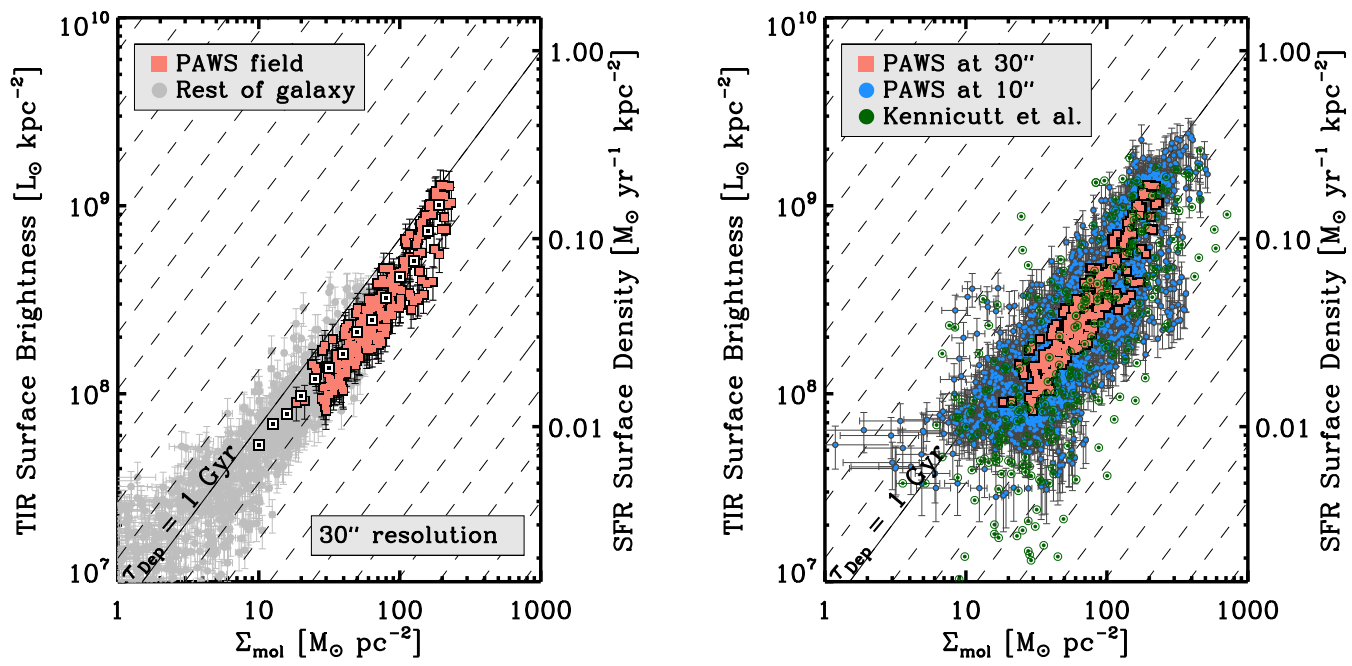

Figure 2. IR-CO scaling relations in M51 over (left) the whole galaxy and (right) in the PAWS field. Diagonal lines indicate a fixed molecular gas depletion times (i.e., a fixed CO-to-IR ratio), spaced by factors of two. Red points in the left panel come from the PAWS field, which we study in this paper. Gray points show the measurements from the PAWS single dish map outside the interferometric survey area. Error bars show statistical uncertainty on individual points. In the left panel, square points show a running mean $\Sigma_{\mathrm{TIR}}$ as a function of $\Sigma_{\text {mol }}$. The weak bowed shape visible in these points may explain some of the discrepancies in the starformation scaling law literature for M51, as different studies focused on different parts of the galaxy. The right panel shows results at both resolutions in the PAWS field and overplots the measurements from Kennicutt et al. (2007), which target 13" apertures on star-forming peaks over a similar area. More variation in the CO-toIR ratio is visible at high resolution, including both high and low $\tau_{\text {Dep }}^{\text {mol }}$ at high $\Sigma_{\text {mol }} \gtrsim 100 M_{\odot} \mathrm{pc}^{-2}$.

and the $13^{\prime \prime}$ measurements of Kennicutt et al. (2007) for selected apertures in green.

Over the whole of M51 (gray points), our data imply a molecular gas depletion time $\tau_{\text {Dep }}^{\text {mol }} \approx 1.5 \mathrm{Gyr}$ with $\sim 0.2$ dex scatter. In the PAWS field (red points), the numbers are about the same, $\tau_{\text {Dep }}^{\text {mol }} \approx 1.7 \mathrm{Gyr}$ with $\sim 0.1$ dex scatter. The median at
$370 \mathrm{pc}$ resolution remains $\tau_{\text {Dep }}^{\text {mol }}=1.6 \mathrm{Gyr}$, but with larger scatter $\sim 0.3$ dex.

This resembles the $\tau_{\text {Dep }}^{\text {mol }} \approx 1-2 \mathrm{Gyr}$ found at the same resolution for a larger sample of similar nearby disk galaxies by Leroy et al. (2013). That study includes M51, but using different data. Our results agree qualitatively with their specific 


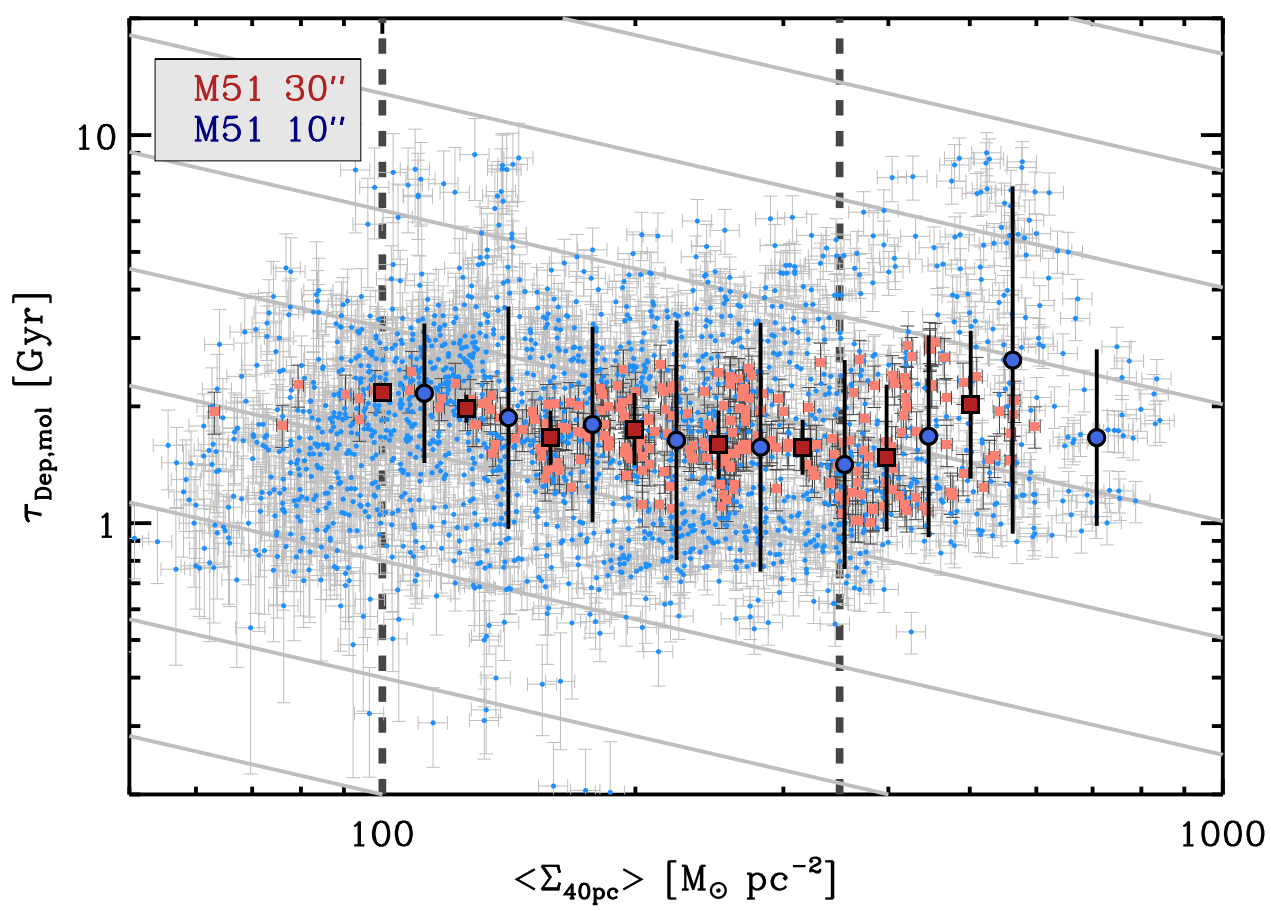

Figure 3. Molecular gas depletion time, $\tau_{\mathrm{Dep}}^{\mathrm{mol}} \equiv \Sigma_{\mathrm{mol}} / \Sigma_{\mathrm{SFR}} \propto I_{\mathrm{CO}} / \Sigma_{\mathrm{TIR}}$, as a function of cloud-scale surface density, $\left\langle\Sigma_{40 \text { pc }}\right\rangle$. Note the difference from Figure 2 , which shows average surface density over large scales. Points here show mass-weighted average $40 \mathrm{pc}$ surface density with a $10^{\prime \prime} \approx 370 \mathrm{pc}$ (blue) and $30^{\prime \prime} \approx 1.1 \mathrm{kpc}$ (red) beam, and so reflect the local cloud-scale surface density. Square points show median $\tau_{\text {Dep }}^{\text {mol }}$ in bins of $\left\langle\Sigma_{40 \text { pc }}\right\rangle$; error bars indicate the rms scatter in the bin. Gray lines show $\tau_{\text {Dep }}^{\text {mol }} \propto\left\langle\Sigma_{40 \text { pc }}\right\rangle^{-0.5}$, which is expected for a constant efficiency per free-fall time and $\left\langle\rho_{40 \mathrm{pc}}\right\rangle \propto\left\langle\Sigma_{40 \mathrm{pc}}\right\rangle$. The dashed vertical lines indicate $\left\langle\Sigma_{40 \mathrm{pc}}\right\rangle=100$ and $350 M_{\odot} \mathrm{pc}^{-2}$. In this range, we observe a mild anticorrelation between $\tau_{\text {Dep }}^{\text {mol }}$ and $\left\langle\Sigma_{40} \mathrm{pc}\right\rangle$, with denser gas forming stars at a higher normalized rate. Above $\left\langle\Sigma_{40 \mathrm{pc}}\right\rangle=350 M_{\odot} \mathrm{pc}^{-2}$, the sense of the correlation between $\left\langle\Sigma_{40 \mathrm{pc}}\right\rangle$ and $\tau_{\text {Dep }}^{\text {mol }}$ shifts and higher surface density gas tends to form stars less effectively.

results for M51, including the presence of high IR-to-CO (low $\tau_{\text {Dep }}^{\text {mol }}$ ) regions in the inner galaxy. Here, the $\mathrm{CO}$ data have much higher signal-to-noise and we use only IR data to trace recent star formation.

The right panel in Figure 2 shows that our data also agree to first order with the measurements by Kennicutt et al. (2007, green, overlapping our blue points). They targeted star-forming peaks with a different measurement strategy, 13" aperture photometry, and use yet another CO map (Helfer et al. 2003) and approach $\Sigma_{\mathrm{SFR}}$, combining Paschen $\alpha$ and $24 \mu \mathrm{m}$ emission.

Over the full area of M51 (left panel), the scaling between IR and $\mathrm{CO}$ exhibits a somewhat "bowed" shape moving from outside the PAWS field (the gray points at low $\Sigma_{\text {mol }}$ ) to the inner disk (red points at high $\Sigma_{\text {mol }}$ ). That is, the slope of the relation is slightly sublinear at low $\Sigma_{\text {mol }}$ and superlinear at high $\Sigma_{\text {mol }}$.

This curvature, which can be seen in the running mean (black-and-white squares) in the left panel, helps explain why different studies targeting M51 have come to apparently contradictory conclusions regarding the slope of the SFR-gas scaling relation (e.g., Liu et al. 2011; Shetty et al. 2013). Those studying the inner part of the galaxy, especially at higher resolution using interferometers, see the superlinear slope evident at high surface densities. Those excluding the inner regions (Bigiel et al. 2008; Shetty et al. 2013) and targeting a wider area find a modestly sublinear slope. That is, given the curved shape of the relation in the left panel of Figure 2, we do not expect a single power law to fit all of M51. Note that this does not explain all of the scatter in the M51 literature, methodological differences including fitting and sampling strategy have also played a role (e.g., see the Appendix in Leroy et al. 2013).
The right panel of Figure 2 shows that, at higher resolution, the IR surface brightness scatters more at fixed $\Sigma_{\text {mol }}$, a result that has been measured before (Blanc et al. 2009; Leroy et al. 2013). The dependence of scatter on scale may be attributed to evolution of individual star-forming regions (e.g., Schruba et al. 2010; Kruijssen \& Longmore 2014), and the 0.3 dex scatter at $\sim 370 \mathrm{pc}$ resolution appears consistent with scatter expected from evolution in the Kruijssen \& Longmore (2014) model.

Our 370 pc measurements may be more stochastic than the $1.1 \mathrm{kpc}$ calculations, but they also allow us to better isolate the physical conditions relevant to star formation. We capture more variation in local cloud populations and are better able to separate the galaxy into distinct regions. Below we find a larger range of ISM structure at $370 \mathrm{pc}$ than $1.1 \mathrm{kpc}$, as well as stronger correlations between environment and ISM structure and distinct results for different dynamical regions.

At 370 pc resolution, we do observe substantial variation in $\Sigma_{\text {TIR }}$ at a given $\Sigma_{\text {mol }}$, including a wide range of $\Sigma_{\text {TIR }}$ at high $\Sigma_{\mathrm{mol}} \gtrsim 100 M_{\odot} \mathrm{pc}^{-2}$. For $\Sigma_{\mathrm{mol}}=30-100 M_{\odot} \mathrm{pc}^{-2}$, the median $\tau_{\text {Dep }}^{\text {mol }} \approx 2$ Gyr with 0.25 dex scatter. For $\Sigma_{\text {mol }}>100 M_{\odot}$

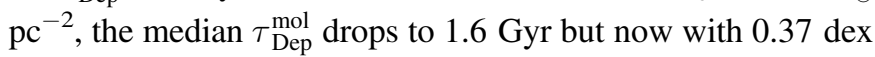
scatter. High IR-to-CO ratios (low $\tau_{\text {Dep }}^{\text {mol }}$ ) are preferentially found at high surface densities, which has helped fuel the result of superlinear power-law scalings for $\Sigma_{\mathrm{SFR}}$ versus $\Sigma_{\text {mol }}$ in M51 (Liu et al. 2011; Momose et al. 2013). However, there are also many lines of sight with high $\Sigma_{\text {mol }}$ and relatively weak IR emission. These unexpected gas-rich, but relatively IR-weak, regions were highlighted by Meidt et al. (2013). They argued that in these regions streaming motions suppress the collapse of gas. 

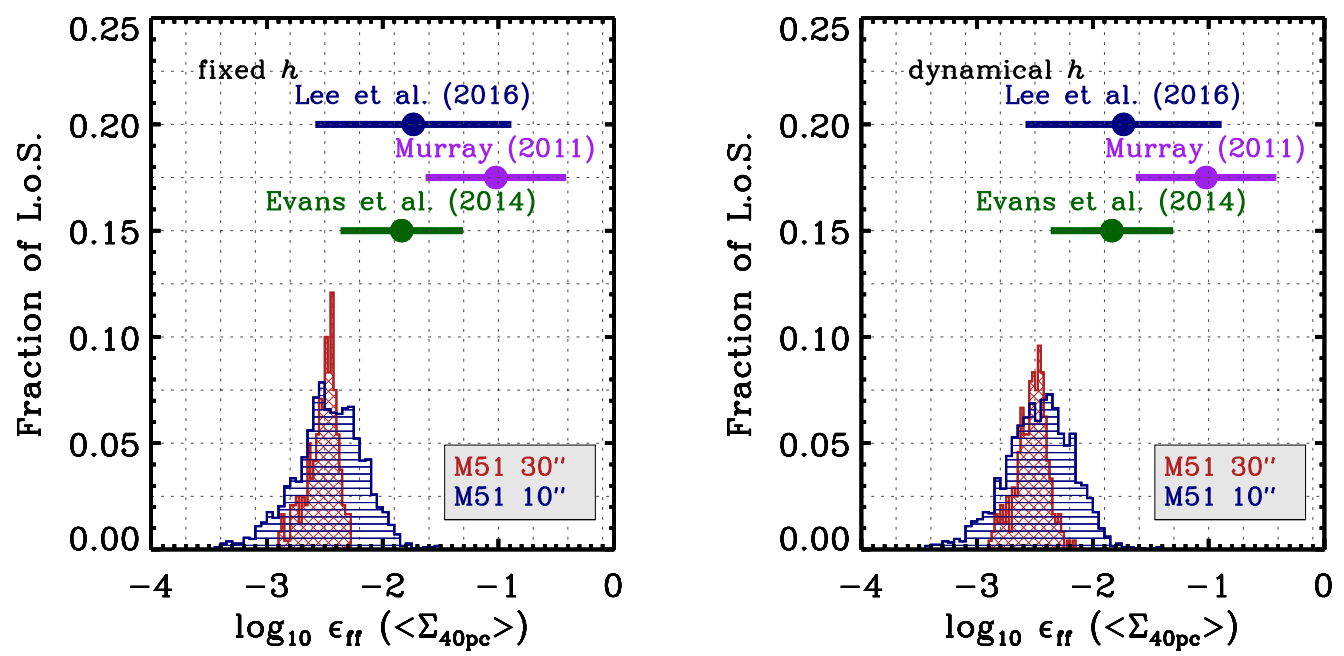

Figure 4. Distributions of the implied efficiency of star formation per free-fall time, $\epsilon_{\mathrm{ff}}\left(\left\langle\Sigma_{40 \mathrm{pc}}\right\rangle\right)$, (left) assuming a fixed line-of-sight depth of $100 \mathrm{pc}$ or (right) a variable depth $\propto b^{-1}=\sigma^{2} / \Sigma$. Both resolutions yield low $\left\langle\epsilon_{\mathrm{ff}, 40 \mathrm{pc}}\right\rangle \approx 0.003-0.0036$, with $\sim 0.3$ dex $(\sim 0.1 \mathrm{dex})$ scatter at $10^{\prime \prime}\left(30^{\prime \prime}\right)$ resolution. The scatter is similar between the two treatments of line-of-sight depth. Colored lines show the median and rms scatter from several Milky Way studies. We suggest that the differences with Lee et al. (2016) and Murray (2011) reflect selection effects, and the difference with Evans et al. (2014) may reflect the influence of extended CO distributions around clouds. In both cases, more work is needed to resolve the discrepancy.

Table 2

Rank Correlation Relating Cloud-scale Structure with $\tau_{\text {Dep }}^{\text {mol }}$ and $\epsilon_{\mathrm{ff}}\left(\left\langle\Sigma_{40 \mathrm{pc}}\right\rangle\right)$

\begin{tabular}{|c|c|c|c|c|c|c|}
\hline Quantity & $\begin{array}{l}\text { Versus } \tau_{\text {Dep }}^{\text {mol }} \\
\text { at } \theta=30^{\prime \prime}\end{array}$ & $\begin{array}{l}\text { Versus } \tau_{\text {Dep }}^{\text {mol }} \\
\text { at } \theta=10^{\prime \prime} \\
\end{array}$ & $\begin{array}{c}\text { Versus } \epsilon_{\mathrm{ff}}\left(\left\langle\sum_{40 \mathrm{pc}}\right\rangle\right) \\
\text { fixed } h \\
\text { at } \theta=30^{\prime \prime}\end{array}$ & $\begin{array}{c}\text { Versus } \epsilon_{\mathrm{ff}}\left(\left\langle\Sigma_{40 \mathrm{pc}}\right\rangle\right) \\
h_{\mathrm{dyn}} \\
\text { at } \theta=30^{\prime \prime}\end{array}$ & $\begin{array}{c}\text { Versus } \epsilon_{\mathrm{ff}}\left(\left\langle\Sigma_{40 \mathrm{pc}}\right\rangle\right) \\
\text { fixed } h \\
\text { at } \theta=10^{\prime \prime}\end{array}$ & $\begin{array}{c}\text { Versus } \epsilon_{\mathrm{ff}}\left(\left\langle\Sigma_{40 \mathrm{pc}}\right\rangle\right) \\
h_{\mathrm{dyn}} \\
\text { at } \theta=10^{\prime \prime}\end{array}$ \\
\hline \multicolumn{7}{|l|}{$\overline{\left\langle\Sigma_{40 \mathrm{pc}}\right\rangle}$} \\
\hline$\ldots$ all data & $-0.14(0.304)$ & $+0.02(0.630)$ & $-0.59(0.000)$ & $-0.78(0.000)$ & $-0.47(0.000)$ & $-0.61(0.000)$ \\
\hline$\ldots 100<\left\langle\Sigma_{40 \mathrm{pc}}\right\rangle<350 \frac{M_{\odot}}{\mathrm{pc}^{-2}}$ & $-0.17(0.307)$ & $-0.14(0.016)$ & $-0.51(0.001)$ & $-0.67(0.000)$ & $-0.15(0.006)$ & $-0.30(0.000)$ \\
\hline$\ldots\left\langle\Sigma_{40 \mathrm{pc}}\right\rangle>350 \frac{M_{\odot}}{\mathrm{pc}^{-2}}$ & $+0.35(0.202)$ & $+0.20(0.089)$ & $-0.51(0.039)$ & $-0.62(0.010)$ & $-0.38(0.002)$ & $-0.45(0.000)$ \\
\hline \multicolumn{7}{|l|}{$\left\langle\sigma_{40} \mathrm{pc}\right\rangle$} \\
\hline$\ldots$ all data & $+0.14(0.278)$ & $+0.26(0.000)$ & $-0.75(0.000)$ & $-0.78(0.000)$ & $-0.61(0.000)$ & $-0.60(0.000)$ \\
\hline$\ldots 100<\left\langle\Sigma_{40 \mathrm{pc}}\right\rangle<350 \frac{M_{\odot}}{\mathrm{pc}^{-2}}$ & $+0.19(0.254)$ & $+0.25(0.000)$ & $-0.69(0.000)$ & $-0.62(0.000)$ & $-0.42(0.000)$ & $-0.33(0.000)$ \\
\hline$\ldots\left\langle\Sigma_{40 \mathrm{pc}}\right\rangle>350 \frac{M_{\odot}}{\mathrm{pc}^{-2}}$ & $+0.84(0.000)$ & $+0.64(0.000)$ & $-0.89(0.000)$ & $-0.84(0.000)$ & $-0.72(0.000)$ & $-0.66(0.000)$ \\
\hline \multicolumn{7}{|l|}{$\left\langle b_{40} \mathrm{pc}\right\rangle$} \\
\hline ...all data & $-0.67(0.000)$ & $-0.42(0.000)$ & $+0.15(0.270)$ & $-0.19(0.145)$ & $+0.13(0.004)$ & $-0.13(0.005)$ \\
\hline$\ldots 100<\left\langle\Sigma_{40 \mathrm{pc}}\right\rangle<350 \frac{M_{\odot}}{\mathrm{pc}^{-2}}$ & $-0.61(0.001)$ & $-0.49(0.000)$ & $+0.30(0.064)$ & $-0.07(0.672)$ & $+0.36(0.000)$ & $+0.09(0.124)$ \\
\hline$\ldots\left\langle\Sigma_{40 \mathrm{pc}}\right\rangle>350 \frac{M_{\odot}}{\mathrm{pc}^{-2}}$ & $-0.79(0.000)$ & $-0.64(0.000)$ & $+0.73(0.001)$ & $+0.56(0.031)$ & $+0.59(0.000)$ & $+0.46(0.000)$ \\
\hline
\end{tabular}

Note. Parenthetical values report the fraction of 1,000 random repairings (accounting for an oversampling factor of 4) that exceed the rank correlation of the true data. They can be read as Monte Carlo $p$ values. $\epsilon_{\mathrm{ff}}\left(\left\langle\Sigma_{40 \mathrm{pc}}\right\rangle\right)$ with "fixed $h$ " assumes a fixed line-of-sight depth of 100 pc. $\epsilon_{\mathrm{ff}}\left(\left\langle\Sigma_{40 \mathrm{pc}}\right\rangle\right)$ with $h_{\mathrm{dyn}}$ uses Equation $(4)$.

Are these region-to-region variations in $\tau_{\text {Dep }}^{\text {mol driven by }}$ changes in the local structure of the gas? In the rest of this section, we explore this idea by comparing $\tau_{\text {Dep }}^{\text {mol }}$ to the local mean $40 \mathrm{pc}$ cloud-scale surface density, velocity dispersion, and gravitational boundedness.

\subsection{Cloud-scale Surface Density and $\tau_{\text {Dep }}^{\text {mol }}$}

All other things being equal, high surface density gas should be denser, with a shorter collapse time, $\tau_{\mathrm{ff}}$. Do the variations in $\tau_{\text {Dep }}^{\text {mol }}$ in Figure 2 arise from changes in the cloud-scale gas density across the galaxy?

Figure 3 tests this expectation, plotting $\tau_{\text {Dep }}^{\text {mol }}$ as a function of $\left\langle\Sigma_{40} \mathrm{pc}\right\rangle$, the mass-weighted cloud-scale surface density in each beam. Table 2 quantifies what we see in the figure, reporting rank correlation coefficients between $\left\langle\Sigma_{40 \text { pc }}\right\rangle$ and $\tau_{\text {Dep }}^{\text {mol }}$ for different ranges of $\left\langle\Sigma_{40 \mathrm{pc}}\right\rangle$.

We do find a weak anticorrelation between $\tau_{\text {Dep }}^{\text {mol }}$ and $\left\langle\Sigma_{40}\right.$ pc $\rangle$ over the range $\left\langle\Sigma_{40 \mathrm{pc}}\right\rangle \approx 100-350 M_{\odot} \mathrm{pc}^{-2}$. Treating $\left\langle\Sigma_{40 \mathrm{pc}}\right\rangle$ as the independent variable yields $\tau_{\text {Dep }}^{\text {mol }} \propto\left\langle\Sigma_{40 \mathrm{pc}}\right\rangle^{-\alpha}$ with $\alpha=0.25-0.35$ over this range. The rank correlation coefficient over this range is only -0.14 , but still statistically significant.

Our simplest expectation would be $\tau_{\text {Dep }}^{\text {mol }} \propto\left\langle\Sigma_{40 \text { pc }}\right\rangle^{-0.5}$. This would be expected if $\rho \propto\left\langle\Sigma_{40}\right.$ pc $\rangle$ (which appears reasonable, see Section 3.2.2), and stars formed from gas with a fixed efficiency per $\tau_{\mathrm{ff}}$. Gray lines in the figure illustrate this slope, which is steeper than the relation that we find. So over 

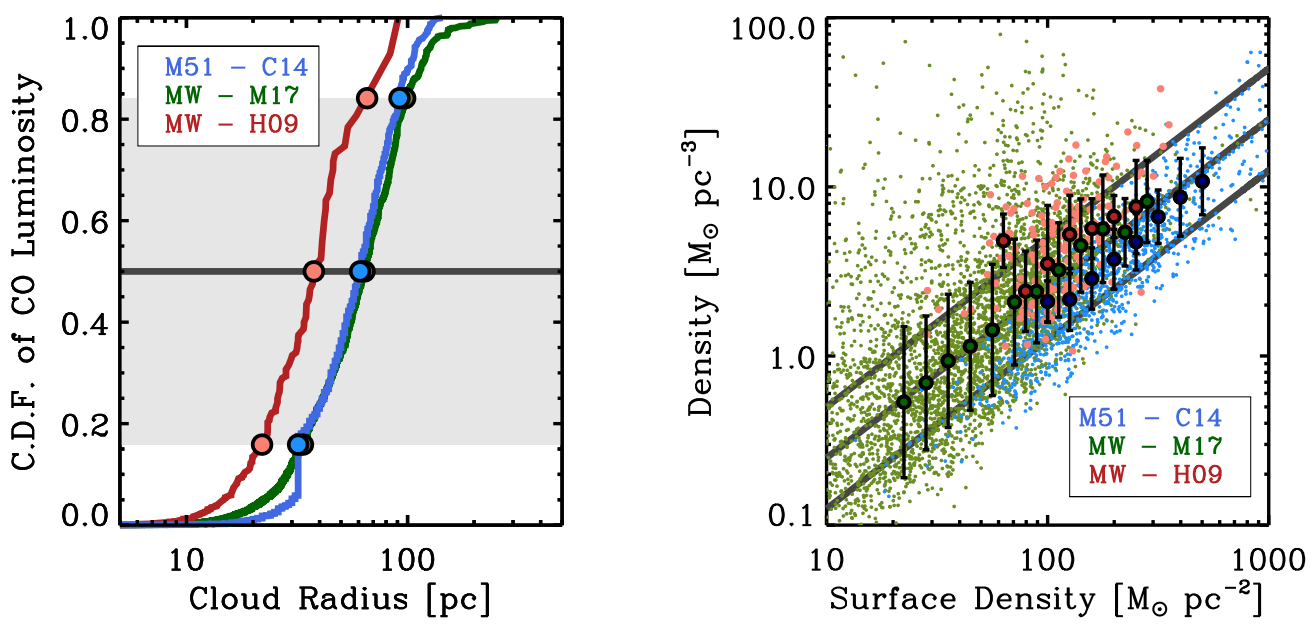

Figure 5. Cloud radius, surface density, and volume density. (Left) The cumulative distribution of CO emission for the M51 GMC catalog of Colombo et al. (2009, C14) and two catalogs of Milky Way (MW) GMCs: the reanalysis of the Solomon et al. (1987) clouds by Heyer et al. (2009, H09) and inner galaxy $r_{\mathrm{gal}}<8.5$ kpc clouds from the full-disk decomposition of Miville-Deschênes et al. (2017, M17). Though there is some offset among the Milky Way measurements, most emission in the Milky Way and M51 catalogs comes from GMCs with $R \gtrsim 30 \mathrm{pc}$ and $R \lesssim 100 \mathrm{pc}$. Along with estimates of the disk thickness in the Milky Way (see Heyer \& Dame 2015) and M51 (see Pety et al. 2013), the plot motivates our fiducial line-of-sight depth $h=100 \mathrm{pc}$ for calculating the free-fall time. (Right) Density of molecular clouds $\rho=M /\left(4 / 3 \pi R^{3}\right)$ as a function of their surface density, $\Sigma=M / \pi R^{2}$, for the same three cloud catalogs. Surface density, our observable, correlates closely with volume density. This supports our treatment of the observable $\Sigma_{40 \mathrm{pc}}$ as a volume density diagnostic. The lines illustrate the relation for left-to-right fixed density $R=15,30$, and $60 \mathrm{pc}$ clouds.

$\left\langle\Sigma_{40} \mathrm{pc}\right\rangle \sim 100-350 M_{\odot} \mathrm{pc}^{-2}$ denser (or at least higher $\left\langle\Sigma_{40 \mathrm{pc}}\right\rangle$ ) gas does appear to form stars at a higher normalized rate, but the efficiency per free-fall time decreases (weakly) as $\left\langle\Sigma_{40 \mathrm{pc}}\right\rangle$ increases.

At higher $\left\langle\Sigma_{40 \mathrm{pc}}\right\rangle>350 M_{\odot} \mathrm{pc}^{-2}, \tau_{\text {Dep }}^{\text {mol }}$ increases with increasing $\left\langle\Sigma_{40 \mathrm{pc}}\right\rangle$, though with large scatter. This leads to the unexpected result pointed out by Meidt et al. (2013) that some of the least efficient star-forming regions in M51 have high cloud-scale molecular gas surface density. We show below that although these regions have high surface densities, they also appear to be less gravitationally bound (higher $\alpha_{\mathrm{vir}}$; Section 3.3.2).

\subsection{Efficiency Per Free-fall Time}

Given a distribution of gas along the line of sight, $\left\langle\Sigma_{40 \mathrm{pc}}\right\rangle$ traces $\rho\left(\left\langle\Sigma_{40} \mathrm{pc}\right\rangle\right)$, the volume density of the gas averaged over the $\theta=40 \mathrm{pc}$ beam. In turn, $\rho\left(\left\langle\Sigma_{40 \mathrm{pc}}\right\rangle\right)$ determines the gravitational free-fall time, $\tau_{\mathrm{ff}}$. Contrasting $\tau_{\mathrm{ff}}$ with the

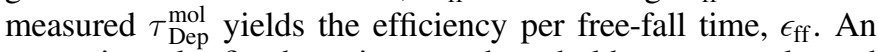
approximately fixed $\epsilon_{\mathrm{ff}}$ is argued to hold across scale and system by, e.g., Krumholz et al. (2012), Krumholz \& McKee (2005), and Krumholz \& Tan (2007). More generally, $\tau_{\text {ff }}$ is taken as the governing timescale for star formation, even when $\epsilon_{\mathrm{ff}}$ is low.

For gas with a depth $h$ along the line-of-sight,

$$
\begin{aligned}
\rho\left(\left\langle\Sigma_{40 \mathrm{pc}}\right\rangle\right) & =\left\langle\Sigma_{40 \mathrm{pc}}\right\rangle / h \\
\tau_{\mathrm{ff}}\left(\left\langle\Sigma_{40 \mathrm{pc}}\right\rangle\right) & =\sqrt{3 \pi /(32 G \rho)}=81 \mathrm{Myr}\left(\frac{\left\langle\Sigma_{40 \mathrm{pc}}\right\rangle}{h_{100 \mathrm{pc}}}\right)^{-0.5} \\
\epsilon_{\mathrm{ff}}\left(\left\langle\Sigma_{40 \mathrm{pc}}\right\rangle\right) & =\tau_{\mathrm{ff}}\left(\left\langle\Sigma_{40 \mathrm{pc}}\right\rangle\right) / \tau_{\text {Dep }}^{\mathrm{mol}}
\end{aligned}
$$

where $h_{100}$ is the depth of the molecular gas layer along the line-of-sight normalized to a fiducial value of $100 \mathrm{pc}$. $\epsilon_{\mathrm{ff}}\left(\left\langle\Sigma_{40 \mathrm{pc}}\right\rangle\right)$ is the efficiency per free-fall time, obtained by contrasting $\tau_{\text {Dep }}^{\text {mol }}$ with $\tau_{\mathrm{ff}}\left(\left\langle\Sigma_{40 \mathrm{pc}}\right\rangle\right)$.
The gray diagonal lines in Figure 3 show $\tau_{\text {Dep }}^{\text {mol }} \propto \Sigma_{\text {mol }}^{-0.5}$. If $h_{100}$ remains fixed, then each of these lines corresponds to a fixed $\epsilon_{\mathrm{ff}}$. In Figure 4, we show the distribution of $\epsilon_{\mathrm{ff}}\left(\left\langle\Sigma_{40 \mathrm{pc}}\right\rangle\right)$ implied by our measurements. We plot results for both working resolutions and show values for a fixed $h=100 \mathrm{pc}$ (left) and $h \propto\left\langle b_{40 \mathrm{pc}}\right\rangle^{-1}$ (right, see explanation Section 3.2.1). We also illustrate the range of $\epsilon_{\mathrm{ff}}$ measured by several Milky Way studies.

We find values of $\epsilon_{\mathrm{ff}}\left(\left\langle\Sigma_{40 \mathrm{pc}}\right\rangle\right)$ that are low in both the absolute sense and relative to theoretical and Milky Way values. We also find $\epsilon_{\mathrm{ff}}\left(\left\langle\Sigma_{40 \mathrm{pc}}\right\rangle\right)$ to vary as a function of environment and the local cloud population. Before discussing this in detail, we motivate our adopted $h$ (Section 3.2.1) and demonstrate that $\left\langle\Sigma_{40 \mathrm{pc}}\right\rangle$ indeed should be a good predictor of $\rho\left(\left\langle\Sigma_{40 \mathrm{pc}}\right\rangle\right)$ (Section 3.2.2).

\subsubsection{What Line-of-sight Depth to Use?}

The depth of the gas layer along the line of sight $h$ affects $\tau_{\mathrm{ff}}\left(\left\langle\Sigma_{40 \mathrm{pc}}\right\rangle\right)$ and so $\epsilon_{\mathrm{ff}}\left(\left\langle\Sigma_{40 \mathrm{pc}}\right\rangle\right)$. We do not observe $h$, but we can make a reasonable estimate. The most common approach is to measure the radius of a GMC on the sky and then assume spherical symmetry. In cloud catalogs for the Milky Way (Heyer et al. 2009; Miville-Deschênes et al. 2017) and M51 (Colombo et al. 2014b), most CO luminosity arises from clouds with radii $\sim 40-60 \mathrm{pc}$. The left panel in Figure 5 shows the distribution of CO luminosity as a function of cloud radius for these three catalogs. The figure shows similar distributions for the Colombo et al. (2014b) M51 catalog and the inner $\left(r_{\text {gal }}<8.5 \mathrm{kpc}\right)$ Milky Way portion of the recent MivilleDeschênes et al. (2017) catalog. In both cases, 68\% of the luminosity comes from clouds with $\sim 30 \mathrm{pc}<R<95 \mathrm{pc}$, with the mid-point for $\mathrm{CO}$ emission $R \sim 60 \mathrm{pc}$. The Heyer et al. (2009) reanalysis of the Solomon et al. (1987) Milky Way clouds (their "A1") yields slightly smaller cloud sizes, $\sim 20 \mathrm{pc}<R<65 \mathrm{pc}$, with median $R \sim 40 \mathrm{pc}$.

Estimates of the thickness of the molecular gas layer in both the Milky Way and M51 yield a similar value. Heyer \& Dame (2015) compile estimates of the thickness of the molecular gas 
disk in the Milky Way (their Figure 6). They find 90-120 pc (FWHM) within the Solar Circle. For M51, Pety et al. (2013) assumed the molecular gas to be in hydrostatic equilibrium. Following Koyama \& Ostriker (2009), they calculated a mean FWHM thickness $\approx 94 \mathrm{pc}$ for the compact portion of the CO disk. If we consider the average density within FWHM $\approx 90 \mathrm{pc}$, then the corresponding $h$ to use in Equation (3) is $h \approx 90 / 0.68=132$ pc.

Thus both estimates of the thickness of the molecular disk and GMC catalogs support our adopted $h \sim 100$ pc. Because $\tau_{\mathrm{ff}} \propto h^{-0.5}$, modest variations in $h$ do not have a large impact on $\epsilon_{\mathrm{ff}}\left(\left\langle\Sigma_{40 \mathrm{pc}}\right\rangle\right)$. Still, we test the impact of varying $h$ by considering the case where the dynamical state of clouds (i.e., the virial parameter) is fixed. Then, $M \propto r \sigma^{2}$ and $r \propto \sigma^{2} / \Sigma=b^{-1}$. The same result applies for gas in a thin disk with only selfgravity. In this case,

$$
h_{\mathrm{dyn}} \equiv 100 \mathrm{pc}\left\langle b_{40 \mathrm{pc}}\right\rangle^{-1} \text {. }
$$

Note that in this situation, where $b$ reflects a changing lineof-sight depth and not a changing dynamical state, $\left\langle b_{40} \mathrm{pc}\right\rangle^{-1}$ and $\left\langle\Sigma_{40 \mathrm{pc}}\right\rangle$ are both linearly proportional to $\left\langle\rho_{40 \mathrm{pc}}\right\rangle$. Then, we expect a similar relation of $\tau_{\text {Dep }}^{\text {mol }}$ to both variables. Below, we show that this is not the case, and our best estimate is that $b$ in fact does reflect a changing dynamical state, not a changing line-of-sight depth. Thus, we consider the case of fixed $h=100 \mathrm{pc}$ to represent our basic result, and use Equation (4) to check the robustness of our conclusions.

\subsubsection{Cloud Surface and Volume Density}

The free-fall time depends on the volume density, $\left\langle\rho_{40 \mathrm{pc}}\right\rangle$, but we observe the surface density, $\left\langle\Sigma_{40 \mathrm{pc}}\right\rangle$. Although it has not been emphasized, these quantities do correlate well in current GMC catalogs. For the Milky Way and M51 catalogs mentioned above, the right panel in Figure 5 shows the volume density of each cloud, $\rho=M /\left(4 / 3 \pi R^{3}\right)$, as a function of its surface density, $\Sigma=M / \pi R^{2}$. Surface and volume density correlate well, with rank correlation coefficients of 0.90 (Colombo et al. 2014a), 0.51 (Heyer et al. 2009), and 0.72 (Miville-Deschênes et al. 2017).

Our mean inferred value for $\epsilon_{\mathrm{ff}}\left(\left\langle\Sigma_{40 \mathrm{pc}}\right\rangle\right)$ does not depend on the assumption that $\left\langle\Sigma_{40 \mathrm{pc}}\right\rangle$ maps perfectly to $\left\langle\rho_{40 \mathrm{pc}}\right\rangle$. Because $\tau_{\text {ff }}$ depends weakly on $h$, it only matters that our adopted lineof-sight depth be roughly correct. But Figure 5 argues that a stronger case holds. The highly observable cloud-scale surface density appears to be a reasonable proxy for the physically important, but hard to directly access volume density. More work on this topic is needed, but the right panel in Figure 5 offers an encouraging sign for extragalactic studies. Cloudscale mapping of $\mathrm{CO}$ surface brightness appears to offer a useful path to probe the mean volume density.

\subsubsection{Low Efficiency Per Free-fall Time}

In Figure $4, \epsilon_{\mathrm{ff}}\left(\left\langle\Sigma_{40 \mathrm{pc}}\right\rangle\right)$ varies between $10^{-3}$ and $10^{-2}$. For both treatments of $h$, the median $\epsilon_{\mathrm{ff}}\left(\left\langle\Sigma_{40 \mathrm{pc}}\right\rangle\right)$ is $3.6 \times 10^{-3}$ with 0.3 dex scatter at $\theta=10^{\prime \prime}$ resolution, and $3.0 \times 10^{-3}$ with 0.11 dex scatter at $\theta=30^{\prime \prime}$ resolution.

These values of $\epsilon_{\mathrm{ff}}\left(\left\langle\Sigma_{40 \mathrm{pc}}\right\rangle\right)$ are low in the absolute sense, with only $0.1 \%-1 \%$ of the gas converted to stars per collapse time. They are also low relative to some expectations from theory and previous work on the Milky Way, though they agree with previous indirect extragalactic estimates of $\epsilon_{\mathrm{ff}}\left(\left\langle\Sigma_{40 \mathrm{pc}}\right\rangle\right)$.
Comparison to estimates at large scales: Our $\epsilon_{\mathrm{ff}}\left(\left\langle\Sigma_{40 \mathrm{pc}}\right\rangle\right) \approx$ $0.3 \%$ agrees with the calculation by Agertz \& Kravtsov (2015), who compared $\tau_{\mathrm{ff}}$ for Galactic GMCs to a typical $\tau_{\text {Dep }}^{\text {mol }}$ for nearby disk galaxies. In a similar vein, our median $\epsilon_{\mathrm{ff}}\left(\left\langle\Sigma_{40 \mathrm{pc}}\right\rangle\right)$ is only a factor of $\sim 2$ lower than the estimate by Murray (2011) of a Milky Way disk-averaged $\epsilon_{\mathrm{ff}} \approx 0.6 \%$.

Observations comparing dense gas, $\mathrm{CO}$, and recent star formation also suggest a low $\epsilon_{\mathrm{ff}}$. García-Burillo et al. (2012) and Usero et al. (2015) observed dense gas tracers, CO, and recent star formation in nearby star-forming galaxies. The combination of these three measurements is sensitive to the density of the gas and to the star formation per unit gas. Thus it depends on $\epsilon_{\mathrm{ff}}$, though in a model-dependent way. GarcíaBurillo et al. (2012) and Usero et al. (2015) argued that a low $\epsilon_{\mathrm{ff}} \approx 0.2 \%$ appears to be required in order for their observations to match the turbulent cloud models of Krumholz \& Tan (2007).

Theoretical values: Our $\epsilon_{\mathrm{ff}}\left(\left\langle\Sigma_{40 \mathrm{pc}}\right\rangle\right) \sim 0.3 \%$ is lower than the $\epsilon_{\mathrm{ff}} \approx 1 \%$ expected at the outer scale of turbulence by Krumholz \& McKee (2005) and Krumholz et al. (2012). Our values are about half of the $\epsilon_{\mathrm{ff}} \approx 0.5 \%$ noted by McKee \& Ostriker (2007). They are also lower than the values commonly adopted by numerical simulations of galaxies (e.g., Agertz \& Kravtsov 2015) or found by simulations of individual starforming regions (e.g., Padoan \& Nordlund 2011).

We note that many of these predictions also depend on the virial parameter (e.g., Padoan \& Nordlund 2002; Krumholz \& McKee 2005), with the Mach number, magnetic support, and type of turbulence also playing a role (e.g., Federrath \& Klessen 2012, 2013). In these cases, matching our observations may be primarily an issue of re-tuning these parameters, though some of these are also constrained by our data (see below).

Comparison to Milky Way results: Our measured $\epsilon_{\mathrm{ff}}\left(\left\langle\Sigma_{40 \mathrm{pc}}\right\rangle\right)$ is significantly lower than the mean $\epsilon_{\mathrm{ff}} \approx 1.5 \%$ found by Evans et al. (2014) for local clouds, and the median $\epsilon_{\mathrm{ff}} \approx 1.8 \%$ found by Lee et al. (2016) based on the MivilleDeschênes et al. (2017) Milky Way GMC catalog and WMAPbased SFRs. It is also much lower than the median $\epsilon_{\mathrm{ff}} \approx 9.5 \%$ found by Murray (2011) for the GMCs associated with the brightest $\sim 32$ star-forming complexes in the Milky Way.

In the case of Murray (2011), this discrepancy is expected. Those clouds were selected based on their association with active star formation, and may have among the highest $\mathrm{SFR} / M_{\text {gas }}$ in the Milky Way. Similarly, the cross-matching of Lee et al. (2016) recovers $\sim 80 \%$ of the ionizing photon flux in their star-forming complexes, but only $\sim 10 \%$ of the GMC mass in the Miville-Deschênes et al. (2017) catalog. Our observations average over the entire life cycle of clouds present in a large averaging beam, and thus can be expected to include the balance of GMC flux. While this has the advantage of better accessing the time-averaged behavior of the gas, it also means that we cannot construct a measurement analogous to Lee et al. (2016) and Murray (2011). In the near future, with a $1^{\prime \prime}$ resolution extinction-robust SFR tracer, we would be able to associate individual clouds with star-forming complexes, and thus potentially access the same dynamical evolution of clouds that leads to the high $\epsilon_{\mathrm{ff}}$ in the Lee et al. (2016) and Murray (2011) results.

Any similar bias toward only star-forming clouds in the Evans et al. (2014) is less clear, but the discrepancy between our "top down" view and the local cloud measurements by Evans et al. (2014) has also been noted before (see Heiderman 

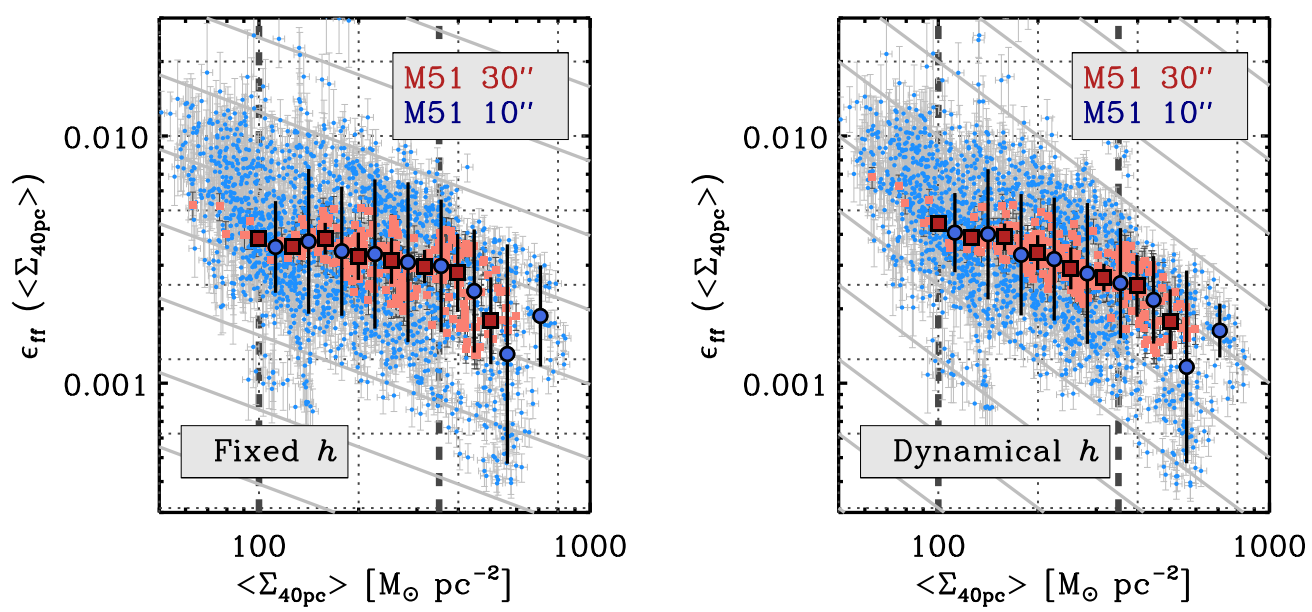

Figure 6. $\epsilon_{\mathrm{ff}}\left(\left\langle\Sigma_{40} \mathrm{pc}\right\rangle\right)$ as a function of $\left\langle\Sigma_{40} \mathrm{pc}\right\rangle$ at $10^{\prime \prime} \approx 370 \mathrm{pc}$ (blue) and $30^{\prime \prime} \approx 1.1 \mathrm{kpc}$ (red) resolution for our two treatments of line-of-sight depth, (left) fixed $h$, our preferred approach and (right) a check using a dynamical estimate of the line-of-sight depth. Gray lines show the expectation if $\tau_{\text {Dep }}^{\text {mol }}$ does not correlate with $\tau_{\mathrm{ff}}$, which is $\epsilon_{\mathrm{ff}}\left(\left\langle\Sigma_{40 \mathrm{pc}}\right\rangle\right) \propto\left\langle\Sigma_{40 \mathrm{pc}}\right\rangle^{-0.5}$ for a fixed $h$ and $\epsilon_{\mathrm{ff}}\left(\left\langle\Sigma_{40 \mathrm{pc}}\right\rangle\right) \propto\left\langle\Sigma_{40 \mathrm{pc}}\right\rangle^{-1}$ for $h \propto b^{-1}=\sigma^{2} / \Sigma$. We observe a weak anticorrelation between $\epsilon_{\mathrm{ff}}\left(\left\langle\Sigma_{40 \mathrm{pc}}\right\rangle\right)$ and $\left\langle\Sigma_{40 \mathrm{pc}}\right\rangle$ at intermediate $\left\langle\Sigma_{40 \mathrm{pc}}\right\rangle \approx 100-350 \mathrm{M}_{\odot} \mathrm{pc}^{-2}$, and then a large drop in $\epsilon_{\mathrm{ff}}\left(\left\langle\Sigma_{40 \mathrm{pc}}\right\rangle\right)$ at higher $\left\langle\Sigma_{40 \mathrm{pc}}\right\rangle>350 \mathrm{M}_{\odot} \mathrm{pc}^{-2}$.

et al. 2010; Lada et al. 2010, 2012). In detail, Evans et al. (2014) find an $\sim 5$ times shorter $\tau_{\text {Dep }}^{\text {mol }}$ for their clouds than we see for large parts of M51. They also find an $\sim 4$ times shorter $\tau_{\mathrm{ff}}$. One plausible explanation for the discrepancy is that Evans et al. (2014) focus on the part of a cloud with $A_{V}>2 \mathrm{mag}$ $\left(\approx 20 M_{\odot} \mathrm{pc}^{-2}\right)$. Including a massive extended envelope or diffuse component might bring both $\tau_{\text {Dep }}^{\text {mol }}$ and $\tau_{\text {ff }}$ into closer agreement with our measured values.

\subsubsection{Possible Systematic Effects}

We argue that most of the discrepancy with Milky Way results can be understood in terms of scales sampled and selection effects. However, several systematic uncertainties could affect our measurement, including our SFR estimate, adopted CO-to- $\mathrm{H}_{2}$ conversion factor, and line-of-sight depth.

Star-formation rate: On average, we would need to be underestimating the SFR of M51 by a factor of 5 to bring our measurements into agreement with the local clouds of Evans et al. (2014). Meanwhile, in the Appendix, we show that many likely biases in $\Sigma_{\text {SFR }}$ would render our TIR-based calculation an overestimate, including any IR cirrus term (Liu et al. 2011; Leroy et al. 2012). Note, however, that Faesi et al. (2014) argue that there may be up to a factor of $\sim 2$ offset between the SFR estimates used in local clouds and the tracers used at larger scales, with the local measurements yielding higher values (see also Lewis et al. 2017). This offset has the right sense, but would have to reach an even larger magnitude to bring our observation into agreement with the local clouds. Also, note that Lee et al. (2016) and Murray (2011) use ionizing photon rates, similar to extragalactic studies.

CO-to- $\mathrm{H}_{2}$ conversion factor: Our adopted $\alpha_{\mathrm{CO}}$ also affects $\epsilon_{\mathrm{ff}}\left(\left\langle\Sigma_{40 \mathrm{pc}}\right\rangle\right)$. We adopt a Galactic conversion factor based on Schinnerer et al. (2010), Colombo et al. (2014a), the calculations in the Appendix, and B. Groves et al. (2017, in preparation). Other work has claimed a lower conversion factor in M51 (see Schinnerer et al. 2010 for a summary). Although evidence from dust, multi-line analysis, and cloud virial masses supports our assumption, the systematic uncertainties in any given determination remain substantial (see Bolatto et al. 2013a). For a lower $\alpha_{\mathrm{CO}}$, we would derive a shorter $\tau_{\text {Dep }}^{\text {mol }}$, a longer $\tau_{\mathrm{ff}}$, and a higher $\epsilon_{\mathrm{ff}}\left(\left\langle\Sigma_{40 \mathrm{pc}}\right\rangle\right)$, with $\epsilon_{\mathrm{ff}}\left(\left\langle\Sigma_{40 \mathrm{pc}}\right\rangle\right) \propto \alpha_{\mathrm{CO}}^{-1.5}$.
$\alpha_{\mathrm{CO}}$ has a stronger effect on $\epsilon_{\mathrm{ff}}\left(\left\langle\Sigma_{40 \mathrm{pc}}\right\rangle\right)$ because it affects both $\tau_{\mathrm{ff}}$ and $\tau_{\text {Dep }}^{\text {mol }}$. Therefore a conversion factor of 0.5 times Galactic would yield $\epsilon_{\mathrm{ff}}\left(\left\langle\Sigma_{40 \mathrm{pc}}\right\rangle\right) \approx 0.85 \%$.

Line-of-sight depth: The adopted line-of-sight depth, $h$, affects $\epsilon_{\mathrm{ff}}\left(\left\langle\Sigma_{40 \mathrm{pc}}\right\rangle\right)$. As emphasized above, our adopted $h$ agrees with both cloud property estimates and modeling of the M51 gas disk. To increase our measured $\epsilon_{\mathrm{ff}}\left(\left\langle\Sigma_{40} \mathrm{pc}\right\rangle\right)$ from $\sim 0.3 \%$ to $\sim 1 \%$, we would need to increase $h$ by an order of magnitude, to $\sim 1 \mathrm{kpc}$. Such a scale height disagrees with the measured cloud properties in M51. A more substantial uncertainty in this direction is the role of any "diffuse" CO disk. Up to $50 \%$ of the CO emission in M51 has been argued to lie in an extended component (Pety et al. 2013). The physical nature of such a component remains unclear, but in the limit that it has a large-scale height and holds half the gas, $\epsilon_{\mathrm{ff}}\left(\left\langle\Sigma_{40 \mathrm{pc}}\right\rangle\right)$ for the compact component could increase by a factor of 2 to $\sim 0.6 \%$. In fact, we do not expect this effect to be so strong, as the bright, compact structures in the combined PdBI $+30 \mathrm{~m}$ map do hold a large fraction of the flux (Leroy et al. 2016), but $10 \mathrm{~s}$ of percent of the CO might lie in such an extended phase. This topic certainly requires more investigation in both the Milky Way and other galaxies.

To summarize, our $\epsilon_{\mathrm{ff}}\left(\left\langle\Sigma_{40 \mathrm{pc}}\right\rangle\right) \sim 0.3 \%-0.36 \%$ does represents our best estimate, though systematic uncertainties could plausibly raise this by a factor of $\sim 2$. Supporting this conclusion, we note that our calculation agrees within a factor of 2 with previous large-scale calculations. Because of the external perspective and averaging approach, we argue that our value represents the correct comparison point for any model aiming to predict a population-averaged $\epsilon_{\mathrm{ff}}\left(\left\langle\Sigma_{40 \mathrm{pc}}\right\rangle\right)$. Cloudby-cloud statistics will need to await future, high-resolution SFR maps.

\subsection{Efficiency Per Free-fall Time, $\tau_{\text {Dep }}^{\text {mol }}$ and Local Gas Properties}

At $370 \mathrm{pc}$ resolution, we find 0.3 dex scatter in $\epsilon_{\mathrm{ff}}\left(\left\langle\Sigma_{40} \mathrm{pc}\right\rangle\right)$, and Figure 3 shows a comparable scatter in $\tau_{\text {Dep }}^{\text {mol }}$. Beyond only estimating $\epsilon_{\mathrm{ff}}\left(\left\langle\Sigma_{40 \mathrm{pc}}\right\rangle\right)$, we aim to understand how the mean gas properties in the beam and the region of the galaxy under consideration influence these two quantities. That is, how much 

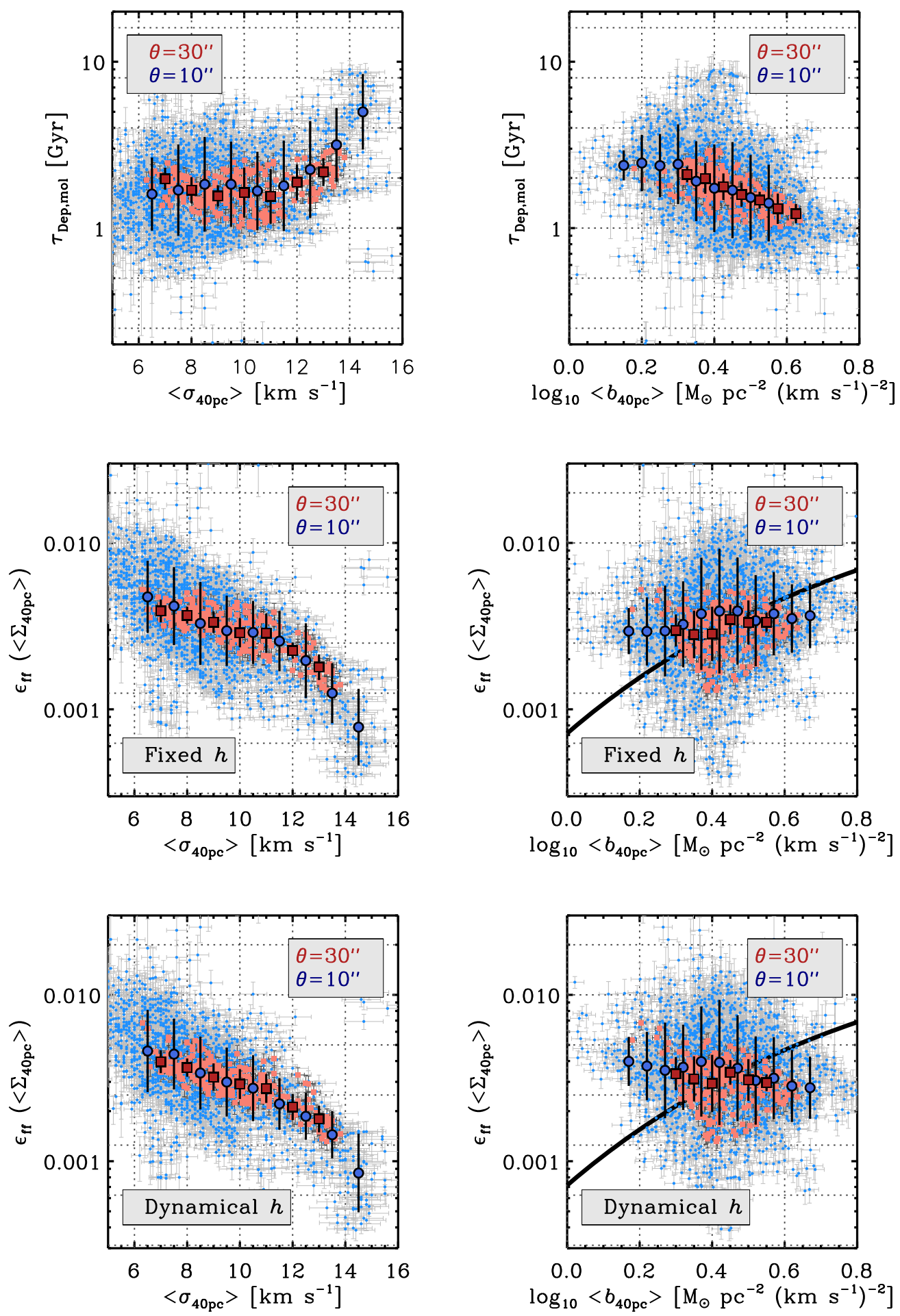

Figure 7. Molecular gas depletion time, $\tau_{\mathrm{Dep}}^{\text {mol }}$ (top row) and efficiency per free-fall time, $\epsilon_{\mathrm{ff}}\left(\left\langle\Sigma_{40 \mathrm{pc}}\right\rangle\right)$ (middle and bottom row) as a function of (left) small-scale velocity dispersion, $\left\langle\sigma_{40}\right.$ pc $\rangle$ and (right) $\left\langle b_{40} \mathrm{pc}\right\rangle \equiv \Sigma / \sigma^{2} \propto \alpha_{\text {vir }}^{-1}$, a tracer of the dynamical state of the gas. (Top left) $\tau_{\text {Dep }}^{\text {mol }}$ shows little relation to $\left\langle\sigma_{40 \mathrm{pc}}\right\rangle$ at low $\lesssim 12 \mathrm{~km} \mathrm{~s}{ }^{-1}$ values. In regions with higher line widths, $>12 \mathrm{~km} \mathrm{~s}^{-1}$, we find high $\tau_{\text {Dep }}^{\text {mol }}$, indicating a low rate of star formation per unit gas mass. (Top right) $\tau_{\text {Dep }}^{\text {mol }}$ anti-correlates with $\left\langle b_{40 \text { pc }}\right.$, indicating a higher rate of star formation per unit gas mass for regions with stronger self-gravity (high $b$, low $\alpha_{\text {vir }}$ ). (Middle and bottom left) the efficiency per free-fall time anti-correlates with the line width across the galaxy, with much lower $\epsilon_{\mathrm{ff}}\left(\left\langle\Sigma_{40 \mathrm{pc}}\right\rangle\right)$ in gas with very large line widths. The same result holds for a fixed line-of-sight depth $h=100 \mathrm{pc}$ or a lineof-sight depth that varies $h \propto b^{-1}$. (Middle and bottom right) $\left\langle\epsilon_{\mathrm{ff}, 40 \mathrm{pc}}\right\rangle$ weakly correlates with $\left\langle b_{40 \mathrm{pc}}\right\rangle$ for the case of a fixed scale height. This becomes a weak anticorrelation if we take $h \propto b^{-1}$; that is, if we assume a fixed dynamical state and use the measured $b$ to infer $h$. The anticorrelation of $\tau_{\text {Dep }}^{\text {mol with }}\left\langle b_{40}\right.$ pc $\rangle$ is much stronger than that with $\left\langle\Sigma_{40 \mathrm{pc}}\right\rangle$; this offers strong, though still indirect, support to the interpretation of the top right panel as a dynamical effect, not a line-of-sight depth effect. The black line in the middle and bottom right panels shows $\epsilon_{\mathrm{ff}}\left(\left\langle\Sigma_{40 \mathrm{pc}}\right\rangle\right) \propto \exp \left(-1.6(5.5 / b)^{0.5}\right)$, approximately the expectation from Padoan et al. (2012), with the normalization chosen to intersect our data.

of this scatter is random and how much results from changes in the local gas properties. Both $\tau_{\text {Dep }}^{\text {mol }}$ and $\epsilon_{\mathrm{ff}}\left(\left\langle\Sigma_{40 \mathrm{pc}}\right\rangle\right)$ are of interest. $\tau_{\text {Dep }}^{\text {mol }}$ captures the SFR per unit gas and represents our most basic observation metric of whether gas in a part of a galaxy is good or bad at forming stars. $\epsilon_{\mathrm{ff}}\left(\left\langle\Sigma_{40 \mathrm{pc}}\right\rangle\right)$ captures the efficiency of star formation relative to direct collapse, with $\tau_{\mathrm{ff}}$ 
representing the most common reference point for current theoretical models.

\subsubsection{Surface Density}

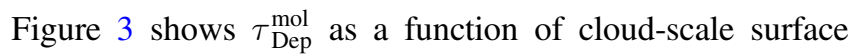
density. Figure 6 shows the corresponding plots for $\epsilon_{\mathrm{ff}}\left(\left\langle\Sigma_{40 \mathrm{pc}}\right\rangle\right)$. As discussed above, $\tau_{\text {Dep }}^{\text {mol }}$ weakly anti-correlates with $\left\langle\Sigma_{40 \mathrm{pc}}\right\rangle$ over the range of $\left\langle\Sigma_{40 \mathrm{pc}}\right\rangle \approx 100-350 \mathrm{M}_{\odot} \mathrm{pc}^{-2}$ and then increases, with large scatter toward higher densities. The observed $\sim-0.3$ slope relating $\tau_{\text {Dep }}^{\text {mol }}$ to $\left\langle\Sigma_{40 \text { pc }}\right\rangle$ is shallower than that expected for a fixed $\epsilon_{\mathrm{ff}}\left(\left\langle\Sigma_{40 \mathrm{pc}}\right\rangle\right)$. As a result, Figure 3 shows $\epsilon_{\mathrm{ff}}\left(\left\langle\Sigma_{40 \mathrm{pc}}\right\rangle\right)$ weakly decreasing with increasing $\left\langle\Sigma_{40 \mathrm{pc}}\right\rangle$ for the fixed $h$ case. Though the slope in the right panel is shallow, Table 2 shows that $\epsilon_{\mathrm{ff}}\left(\left\langle\Sigma_{40 \mathrm{pc}}\right\rangle\right)$ does correlate with $\left\langle\Sigma_{40 \mathrm{pc}}\right\rangle$ over this range with good significance.

This trend in $\epsilon_{\mathrm{ff}}\left(\left\langle\Sigma_{40 \mathrm{pc}}\right\rangle\right)$ is weak compared to the large scatter until $\left\langle\Sigma_{40 \mathrm{pc}}\right\rangle>350 M_{\odot} \mathrm{pc}^{-2}$, at which point $\epsilon_{\mathrm{ff}}\left(\left\langle\Sigma_{40 \mathrm{pc}}\right\rangle\right)$ drops precipitously. The high $\tau_{\text {Dep }}^{\text {mol }}$ at high $\left\langle\Sigma_{40 \text { pc }}\right\rangle$ in Figure 3 corresponds to even lower $\epsilon_{\mathrm{ff}}\left(\left\langle\Sigma_{40 \mathrm{pc}}\right\rangle\right)$. Thus the very high surface density parts of M51 (the inner arms; see Meidt et al. 2013, and next section) are significantly less efficient than the rest of the galaxy at forming stars relative to the expectation for direct collapse $\left(\tau_{\mathrm{ff}}\right)$. The most extreme values in Figure 6 reach $<0.1 \%$, though $\sim 0.2 \%$ represents a more typical $\epsilon_{\mathrm{ff}}\left(\left\langle\Sigma_{40 \mathrm{pc}}\right\rangle\right)$ at these high $\left\langle\Sigma_{40 \mathrm{pc}}\right\rangle$.

The right panel adopts our alternate treatment of $h$ (Equation (4)). The main difference from the left panel is a stronger anticorrelation between $\epsilon_{\mathrm{ff}}\left(\left\langle\Sigma_{40 \mathrm{pc}}\right\rangle\right)$ and $\left\langle\Sigma_{40 \mathrm{pc}}\right\rangle$ at intermediate surface densities (see Table 2). The left panel represents our best estimate, but the consistency between the two suggests that our qualitative results are robust: there is some anticorrelation between $\epsilon_{\mathrm{ff}}\left(\left\langle\Sigma_{40 \mathrm{pc}}\right\rangle\right)$ and $\left\langle\Sigma_{40 \mathrm{pc}}\right\rangle$ at intermediate densities and even lower $\epsilon_{\mathrm{ff}}\left(\left\langle\Sigma_{40 \mathrm{pc}}\right\rangle\right)$ at high $\left\langle\Sigma_{40 \text { pc }}\right\rangle$.

Note that the axes in Figure 6 are correlated because $\tau_{\mathrm{ff}} \propto\left\langle\Sigma_{40 \mathrm{pc}}\right\rangle^{-0.5}$ in both panels. This built-in correlation is stronger in the right panel because for our dynamical scale height (Equation (4)) $h \propto\left\langle\Sigma_{40 \mathrm{pc}}\right\rangle^{-1}$. The statistical uncertainty in $\left\langle\Sigma_{40} \mathrm{pc}\right\rangle$ is small, $\sim 5 \%$, and therefore we do not expect correlated noise to affect the results much. The larger issue is that if $\tau_{\text {Dep }}^{\text {mol }}$ and $\tau_{\mathrm{ff}}$ are unrelated, then $\epsilon_{\mathrm{ff}}\left(\left\langle\Sigma_{40 \mathrm{pc}}\right\rangle\right) \propto$ $\left\langle\Sigma_{40 \mathrm{pc}}\right\rangle^{-0.5}$ for fixed $h$ by construction. That is, the null hypothesis that $\tau_{\mathrm{ff}}$ is not a governing timescale for star formation, we expect an anticorrelation in Figure 6. This does not invalidate the measurement, but should be kept in mind when interpreting the plot.

\subsubsection{Velocity Dispersion}

Surface density and volume density are not the only relevant properties of the gas. In a turbulence-regulated view of star formation, clouds with a high Mach number have a wider density distribution and include more dense gas (Padoan \& Nordlund 2002). The Mach number also affects the critical density for the onset of star formation (e.g., Krumholz \& McKee 2005), with a higher threshold density expected for higher Mach numbers.

Specific predictions differ from model to model (see Federrath \& Klessen 2012), but most models predict an increase in $\epsilon_{\mathrm{ff}}$ for high $\mathcal{M}$. If the temperature does not vary strongly across M51, and if the line widths that we observe are primarily turbulent in nature, then $\left\langle\sigma_{40 \mathrm{pc}}\right\rangle$ should reflect the turbulent Mach number. In this case, if the turbulent models are right, then we would expect $\epsilon_{\mathrm{ff}}\left(\left\langle\Sigma_{40 \mathrm{pc}}\right\rangle\right)$ to correlate with $\left\langle\sigma_{40} \mathrm{pc}\right\rangle$.

We test these expectations in the left panels of Figure 7 . We plot $\tau_{\text {Dep }}^{\text {mol }}$ (top) and $\epsilon_{\mathrm{ff}}\left(\left\langle\Sigma_{40 \mathrm{pc}}\right\rangle\right)$ (middle and bottom) as a function of $\left\langle\sigma_{40 \mathrm{pc}}\right\rangle$. We do not observe a significant correlation between $\left\langle\sigma_{40 \mathrm{pc}}\right\rangle$ and $\tau_{\text {Dep }}^{\text {mol }}$ at intermediate values of $\left\langle\sigma_{40 \mathrm{pc}}\right\rangle \approx 6-12 \mathrm{~km} \mathrm{~s}^{-1}$. At high values of $\left\langle\sigma_{40} \mathrm{pc}\right\rangle$, we tend to find higher $\tau_{\text {Dep }}^{\text {mol }}$. That is, where $\left\langle\sigma_{40 \mathrm{pc}}\right\rangle$ appears high, gas appears inefficient at forming stars.

Normalizing by the free-fall time, the middle and bottom left panels of Figure 7 show a steady decrease in $\epsilon_{\mathrm{ff}}\left(\left\langle\Sigma_{40 \mathrm{pc}}\right\rangle\right)$ with increasing $\left\langle\sigma_{40 \mathrm{pc}}\right\rangle$. The decline becoming steeper at high $\left\langle\sigma_{40 \mathrm{pc}}\right\rangle$. The trend remains qualitatively the same for both treatments of line-of-sight depth. This anticorrelation is unexpected in turbulent theories. It suggests that the primary impact of the measured line width, whatever its origin, is to offer increased support against collapse rather than to increase the abundance of dense gas.

Based on modeling the velocity field, Meidt et al. (2013) and Colombo et al. (2014b) suggested that the line widths in M51 include substantial contributions from unresolved bulk motions. In this case, $\left\langle\sigma_{40 \mathrm{pc}}\right\rangle$ may instead indicate the strength shearing or streaming motions, which can play a key role in suppressing star formation (Meidt et al. 2013). This seems very likely to explain the long depletion times at high $\left\langle\sigma_{40 \mathrm{pc}}\right\rangle\left(\gtrsim 12 \mathrm{~km} \mathrm{~s}^{-1}\right)$.

At lower $\left\langle\sigma_{40 \mathrm{pc}}\right\rangle$ the picture is less clear. M51 obeys the standard GMC scaling relations Colombo et al. (2014a), including when analyzed beam-by-beam Leroy et al. (2016), so we do expect that over most of the galaxy $\left\langle\sigma_{40}\right.$ pc $\rangle$ reflects the turbulent line width to a reasonable degree (though see S. Meidt et al. 2017, submitted). In this case, Figure 7 presents a result not expected in turbulent theory: that high line width implies a low efficiency per free-fall time. Making similar measurements in other galaxies will help illuminate whether this effect is general or indeed driven by the large-scale dynamics of M51.

\subsubsection{Dynamical State}

Neither the surface density nor the line width exist in a vacuum. Instead, they correlate (see Leroy et al. 2016), so that the high $\tau_{\text {Dep }}^{\text {mol }}$, high $\left\langle\sigma_{40}\right.$ pc $\rangle$ points in Figure 7 are also the high surface density points seen above. Their balance, $\Sigma / \sigma^{2}$, reflects the relative strength of the gravitational potential and the kinetic energy of the gas. In almost any view of star formation, a higher degree of self-gravity will render gas better at forming stars. In turbulent theories, this manifests as a dependence of $\epsilon_{\mathrm{ff}}$ on the virial parameter (e.g., Krumholz \& McKee 2005), or the closely related ratio of free-fall time to crossing time (e.g., Padoan \& Nordlund 2011).

We capture the balance of gravitational potential and kinetic energy via $\left\langle b_{40 \mathrm{pc}}\right\rangle \equiv\left\langle\Sigma_{40 \mathrm{pc}}\right\rangle /\left\langle\sigma_{40 \mathrm{pc}}\right\rangle^{2} \propto \mathrm{UE} / \mathrm{KE} \propto \alpha_{\text {vir }}^{-1}$. When $\left\langle b_{40} \mathrm{pc}\right\rangle$ is high, the surface density is high relative to the line width and the gas is more tightly bound; when $\left\langle b_{40 \mathrm{pc}}\right\rangle$ is low it has a large kinetic energy compared to its inferred potential.

The right panels in Figure 7 show $\tau_{\text {Dep }}^{\text {mol }}$ (top) and $\epsilon_{\mathrm{ff}}\left(\left\langle\Sigma_{40 \mathrm{pc}}\right\rangle\right)$ (middle and bottom) as a function of $\left\langle b_{40 \mathrm{pc}}\right\rangle$. We observe a significant anticorrelation between $\tau_{\text {Dep }}^{\text {mol }}$ and 

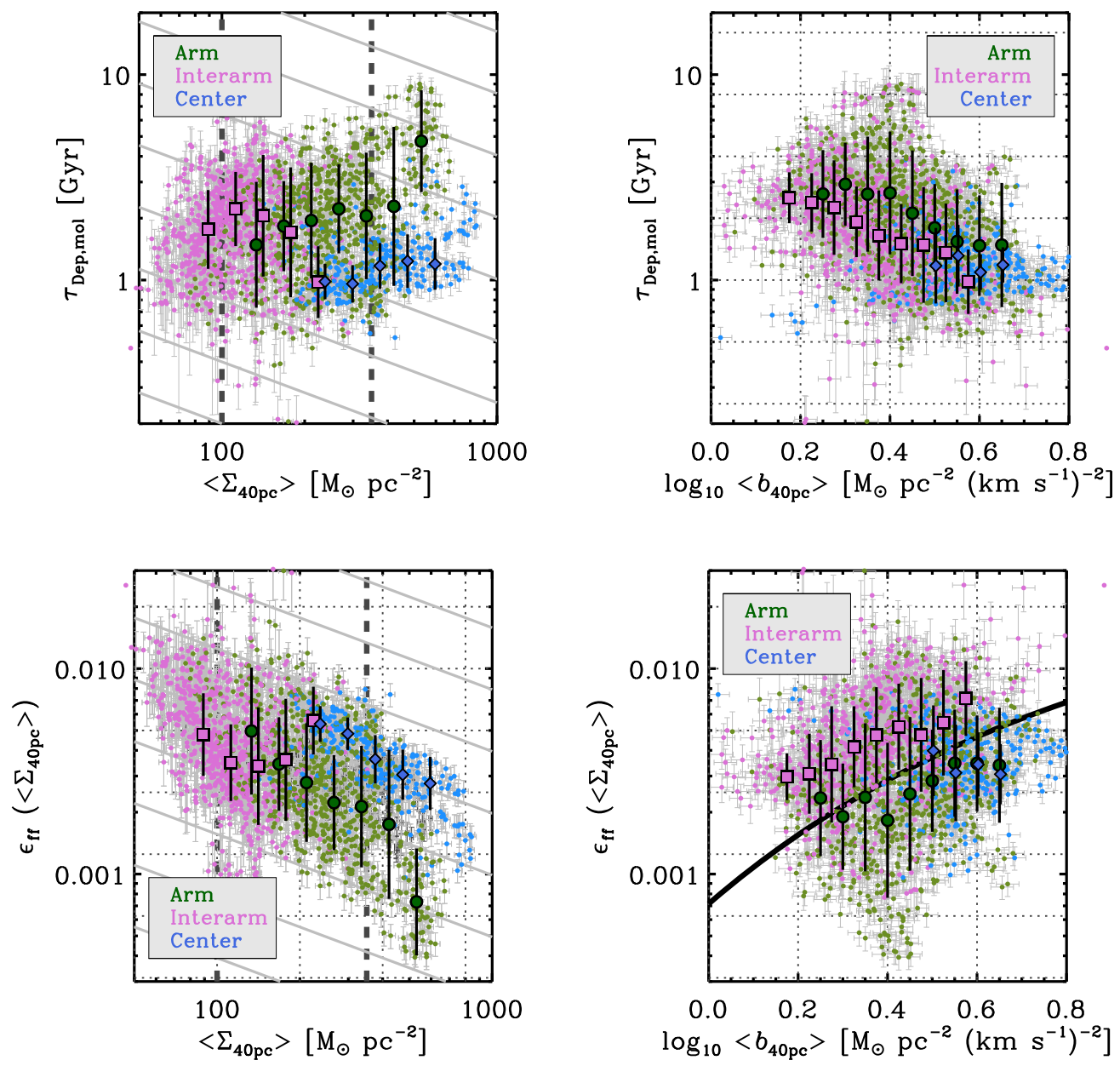

Figure 8. (Top) Molecular gas depletion time, $\tau_{\text {Dep }}^{\text {mol }}$, and (bottom) efficiency per free-fall time, $\epsilon_{\mathrm{ff}}\left(\left\langle\Sigma_{40 \mathrm{pc}}\right\rangle\right)$, for $h=100 \mathrm{pc}$ as a function of $\left\langle\Sigma_{40 \mathrm{pc}}\right\rangle$ and $\left\langle b_{40 \mathrm{pc}}\right\rangle$ (as in Figures 3 and 7 ) all at $10^{\prime \prime} \approx 370 \mathrm{pc}$ resolution, but now plotting measurements from the arm (green), interarm (purple), and central (blue) regions of the galaxy separately. Gray lines again show the expectation for fixed efficiency per free-fall time. The galaxy separates by region in the $\tau_{\text {Dep }}^{\text {mol }}$ vs. $\left\langle\Sigma_{40}\right.$ pc $\rangle$ diagram. The behavior of different regions appears more similar in $\tau_{\text {Dep }}^{\text {mol }}$ vs. $b$, consistent with the dynamical state of the gas explaining most of the observed variations in $\tau_{\text {Dep }}^{\text {mol }}$ Considering $\epsilon_{\mathrm{ff}}\left(\left\langle\Sigma_{40 \mathrm{pc}}\right\rangle\right)$ as a function of $\left\langle b_{40 \mathrm{pc}}\right\rangle$ (bottom right panel), individual regions show a larger indication than the galaxy as a whole for an expected positive correlation between $\epsilon_{\mathrm{ff}}\left(\left\langle\Sigma_{40 \mathrm{pc}}\right\rangle\right)$ and $b \equiv \Sigma / \sigma^{2} \propto \alpha_{\text {vir }}^{-1}$. The black line in the bottom right panels shows $\epsilon_{\mathrm{ff}}\left(\left\langle\Sigma_{40 \mathrm{pc}}\right\rangle\right) \propto \exp \left(-1.6(5.5 / b)^{0.5}\right)$, approximately the expectation from Padoan et al. (2012), with the normalization chosen to intersect our data.

$\left\langle b_{40} \mathrm{pc}\right\rangle$. The sense of this anticorrelation is that more bound gas (high $b$ )-equivalently, gas with a high ratio of $\tau_{\mathrm{ff}}$ to $\tau_{\text {cross }}$-forms stars at a high rate per unit gas mass (low $\tau_{\text {Dep }}^{\text {mol }}$ ). The strength of the anticorrelation is striking given the weak and inconsistent relationships between $\tau_{\text {Dep }}^{\text {mol }}$ and $\left\langle\Sigma_{40 \mathrm{pc}}\right\rangle$ or $\left\langle\sigma_{40 \mathrm{pc}}\right\rangle$. A fit to the data treating $\left\langle b_{40 \mathrm{pc}}\right\rangle$ as the independent variable and using the form $\tau_{\text {Dep }}^{\text {mol }} \propto\left\langle b_{40} \text { pc }\right\rangle^{-\alpha}$ gives $\alpha=-0.8$ to -1.0 , with the range depending moderately on the resolution and approach used to determine the best-fitting relationship.

$\left\langle b_{40} \mathrm{pc}\right\rangle$ Probably does reflect dynamical state: As discussed above, $b$ can be interpreted in two ways. If the line-of-sight depth remains constant, then $b$ traces the dynamical state of the gas, $b \propto \alpha_{\text {vir }}^{-1}$. Alternatively, if the dynamical state of the gas remains fixed, e.g., if all gas is marginally bound or virialized, then $b$ indicates the line-of-sight depth, with $h \propto b^{-1}$.

Figure 7 offers a strong, if indirect, argument that variations in $\left\langle b_{40} \mathrm{pc}\right\rangle$ do mainly reflect changes in the dynamical state. Compare the clear, steep anticorrelation between $\tau_{\text {Dep }}^{\text {mol }}$ and $b$ to the weak relation between $\tau_{\text {Dep }}^{\text {mol }}$ and $\left\langle\Sigma_{40 \text { pc }}\right\rangle$ seen in Figure 3. If the density of gas is the only variably relevant to star formation, then we would expect the two figures to show similar relations because $\rho \propto \Sigma / h$. Instead, only $b$ shows a strong anticorrelation with $\tau_{\text {Dep }}^{\text {mol }}$. Furthermore, the slope of the anticorrelation is approximately -0.8 to -1.0 , steeper than the slope of -0.5 expected from only $\tau_{\text {Dep }}^{\text {mol }} \propto \rho^{-0.5}$.

$\left\langle b_{40 \mathrm{pc}}\right\rangle$ and $\epsilon_{\mathrm{ff}}\left(\left\langle\Sigma_{40 \mathrm{pc}}\right\rangle\right)$ : The importance of $b \sim \alpha_{\mathrm{vir}}^{-1}$ has been highlighted by Padoan et al. (2012) and others (e.g., Krumholz \& McKee 2005). Gas with a lower virial parameter and a higher $\mathrm{UE} / \mathrm{KE}$ or $b$ is expected to be better at forming stars. Our result broadly supports these expectations.

Turbulent theories often predict an impact of $\alpha_{\text {vir }}$ on the efficiency per free-fall time, however, not the gas depletion time. The middle and bottom right panels of Figure 7 show $\epsilon_{\mathrm{ff}}\left(\left\langle\Sigma_{40 \mathrm{pc}}\right\rangle\right)$ as a function of $\left\langle b_{40 \mathrm{pc}}\right\rangle$. There, the impact of $b$ is less clear. Formally, we find a weak but significant positive correlation if we hold $h$ fixed, so that $\epsilon_{\mathrm{ff}}\left(\left\langle\Sigma_{40} \mathrm{pc}\right\rangle\right)$ is higher with higher $\left\langle b_{40}\right.$ pc $\rangle$. But the figure shows that this is a modest effect, and the trend reverses if we allow $h$ to vary.

Padoan et al. (2012) predict $\epsilon_{\mathrm{ff}} \approx 0.5 \exp \left(-1.6 \tau_{\mathrm{ff}} / \tau_{\text {cross }}\right) \propto$ $\exp \left(-1.6 b^{-0.5}\right)$. We show a modified version of this prediction as a black line in the figures. We take $\alpha_{\text {vir }}=5.5 / b$, which is 

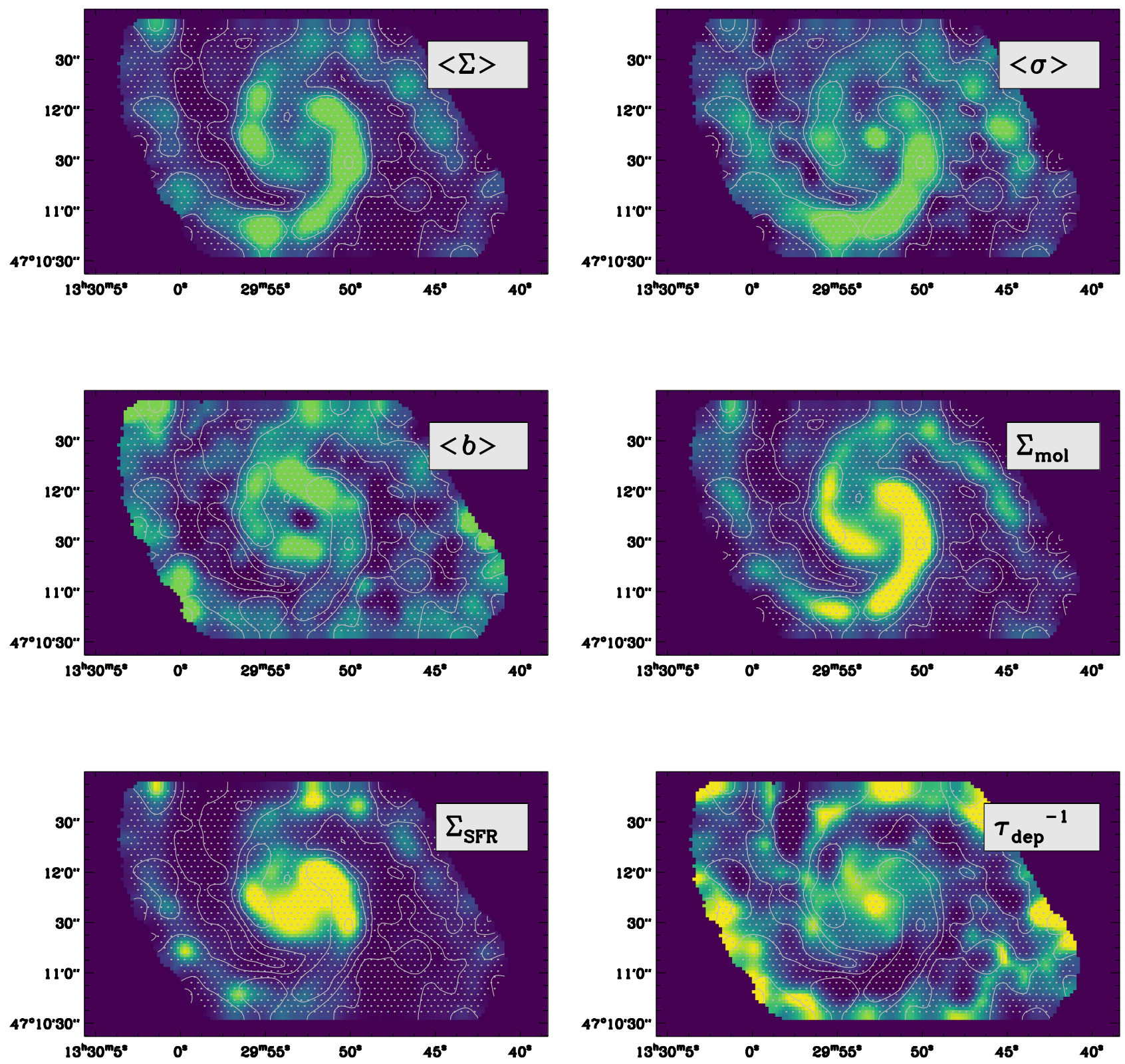

Figure 9. Maps of $\left\langle\Sigma_{40 \mathrm{pc}}\right\rangle,\left\langle\sigma_{40 \mathrm{pc}}\right\rangle,\left\langle b_{40 \mathrm{pc}}\right\rangle, \Sigma_{\mathrm{mol}}, \Sigma_{\mathrm{TIR}} \propto \Sigma_{\mathrm{SFR}}$, and $\left(\tau_{\mathrm{Dep}}^{\mathrm{mol}}\right)^{-1} \equiv \Sigma_{\mathrm{SFR}} / \Sigma_{\text {mol }}$ at $10^{\prime \prime} \approx 370 \mathrm{pc}$ resolution. The same contours of $\left\langle\Sigma_{40 \mathrm{pc}}\right\rangle$ appear in all of the images, and all images are stretched to show a linear stretch covering the middle $95 \%$ of the data. The star-forming ring and the outer spiral arm regions show high rates of star formation per unit gas mass, and also high $b$. The inner spiral arms show high $\Sigma_{\text {mol }}$ and $\left\langle\Sigma_{40 \text { pc }}\right\rangle$, but even higher $\left\langle\sigma_{40 \text { pc }}\right\rangle$, leading to a low $b$ and comparatively weak star formation.

appropriate for clouds with $R \sim 60 \mathrm{pc}$, and set the normalization to pass through our data. Similar to the results of Lee et al. (2016) in the Milky Way, the Padoan et al. (2012) prediction does not seem to capture the full set of physics at play in our data. Though we show in the next section that it offers a better match to the data for individual dynamical regions.

\subsection{Relation to Galaxy Structure}

M51 exhibits strong spiral and radial structure. Large-scale gas flows have been linked to the ability of M51's gas to form stars (e.g., Koda et al. 2009) and to the suppression of star formation by streaming motions (e.g., Meidt et al. 2013). Figure 8 shows how $\tau_{\text {Dep }}^{\text {mol }}$ and $\epsilon_{\mathrm{ff}}\left(\left\langle\Sigma_{40 \mathrm{pc}}\right\rangle\right)$ vary with $\left\langle\Sigma_{40 \mathrm{pc}}\right\rangle$ and $\left\langle b_{40 \mathrm{pc}}\right\rangle$ region-by-region. Here, we color the points according to the dynamical region from which most of the $\mathrm{CO}$ emission in the beam originates. We show the twodimensional distributions of $\left\langle\Sigma_{40 \mathrm{pc}}\right\rangle,\left\langle\sigma_{40 \mathrm{pc}}\right\rangle,\left\langle b_{40} \mathrm{pc}\right\rangle, \Sigma_{\mathrm{mol}}$, $\Sigma_{\mathrm{SFR}}$, and $\left(\tau_{\mathrm{Dep}}^{\mathrm{mol}}\right)^{-1}$ in Figure 9.

Figure 8 shows $\tau_{\text {Dep }}^{\text {mol }}$ as a function of $\left\langle\Sigma_{40}\right.$ pc $\rangle$ for arm (green), interarm (purple), and central (blue) parts of the galaxy. As previously shown by Koda et al. (2009), Hughes et al. (2013b), and (Colombo et al. 2014a), the cloud-scale surface density increases dramatically, moving from the interarm to the arm region. The center of the galaxy exhibits high gas surface densities.

Although the arms concentrate molecular gas, we do not

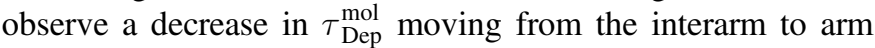


regions. Combining the arm and interarm regions, $\tau_{\text {Dep }}^{\text {mol }}$ remains approximately constant as a function of surface density until it rises at the highest values of $\left\langle\Sigma_{40 \mathrm{pc}}\right\rangle$. This is the apparent suppression of star formation-despite high surface densitiesobserved in the arms by Meidt et al. (2013). These observations are also consistent with the observation by Foyle et al. (2010)

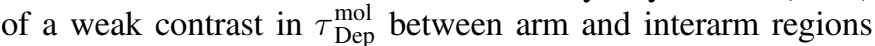
in M51.

The inner part of M51 has high $\left\langle\Sigma_{40 \text { pc }}\right\rangle$, similar to that found in the spiral arms. Here, however, the high surface densities are accompanied by low $\tau_{\text {Dep }}^{\text {mol }}$. As a result, in the top left panel of Figure 8 , the points at high $\left\langle\Sigma_{40 \text { pc }}\right\rangle$ separate in $\tau_{\text {Dep }}^{\text {mol according }}$ to the region from which they arise. As Figure 9 shows, many of the lowest $\tau_{\text {Dep }}^{\text {mol }}$ arise from the star-forming ring of the galaxy. These correspond to the high $\Sigma_{\text {SFR }}$ points in the scaling relations in Figure 2. The few points at the galaxy center, in which AGN contamination (Querejeta et al. 2016) and beam smearing (e.g., S. Meidt et al. 2017, in preparation) contribute most, has little effect on the overall trend.

The top right panel of Figure 8 shows that, although the parts of the galaxy separate in $\tau_{\text {Dep }}^{\text {mol }}$ versus $\left\langle\Sigma_{40 \mathrm{pc}}\right\rangle$ space, they overlap much better when $\tau_{\text {Dep }}^{\text {mol }}$ is plotted as a function of $\left\langle b_{40 \mathrm{pc}}\right\rangle$. That is, the long depletion times observed at high $\left\langle\Sigma_{40 \text { pc }}\right\rangle$ in the arms appear to be there because that gas has low $\left\langle b_{40 \mathrm{pc}}\right\rangle$, i.e., it appears weakly gravitationally bound. We observe an anticorrelation between $\tau_{\text {Dep }}^{\text {mol }}$ and $b$ in both the arm and interarm regions. The central region, which has the lower $\tau_{\text {Dep }}^{\text {mol }}$, also has the strongest self-gravity, traced by $b$.

We do observe an offset between the median $\tau_{\text {Dep }}^{\text {mol }}$ in the arm and interarm region at fixed $b$. At the same $\left\langle b_{40} \mathrm{pc}\right\rangle$, points in the arms have typically $0.13 \mathrm{dex}(\sim 35 \%)$ longer $\tau_{\text {Dep }}^{\text {mol }}$. This could reflect evolutionary effects on scales larger than our averaging beam. For example, Schinnerer et al. (2017) show the formation of stars along spurs displaced downstream from the arms. Or it could be driven additional suppression of star formation in the arms by dynamical effects not captured by $\left\langle b_{40 \mathrm{pc}}\right\rangle$ (Meidt et al. 2013). Alternatively, it could reflect a lower filling fraction in the interarm region, so that beam dilution affects the interarm points more, lowering $b$ relative to its true value. It could also reflect a low level bias in our SFR tracers, which affects the lower-magnitude $\Sigma_{\mathrm{SFR}}$ in the interarm more than in the arm.

When recast from $\tau_{\text {Dep }}^{\text {mol }}$ to $\epsilon_{\mathrm{ff}}\left(\left\langle\Sigma_{40 \mathrm{pc}}\right\rangle\right)$ as a function of $\left\langle\Sigma_{40 \text { pc }}\right\rangle$ (bottom row), the galaxy again separates. Here the arms appear as outliers. They show low $\epsilon_{\mathrm{ff}}\left(\left\langle\Sigma_{40 \mathrm{pc}}\right\rangle\right)$, significantly lower than the interarm region or the center. That is, given the high surface densities in the arms, we would expect collapse to proceed quickly. But the observed $\tau_{\text {Dep }}^{\text {mol }}$ does not support this expectation. The contrast between these low $\epsilon_{\mathrm{ff}}\left(\left\langle\Sigma_{40 \mathrm{pc}}\right\rangle\right)$ in the arms and the higher values in the interarm regions drive the anticorrelation between $\epsilon_{\mathrm{ff}}\left(\left\langle\Sigma_{40 \mathrm{pc}}\right\rangle\right)$ and $\left\langle\Sigma_{40 \text { pc }}\right\rangle$ observed across the whole galaxy.

The bottom right panel of Figure 8 shows $\epsilon_{\mathrm{ff}}\left(\left\langle\Sigma_{40 \mathrm{pc}}\right\rangle\right)$ as a function of $\left\langle b_{40} \mathrm{pc}\right\rangle$ region-by-region. When we considered the whole galaxy (Figure 7), only a weak correlation related $\epsilon_{\mathrm{ff}}\left(\left\langle\Sigma_{40 \mathrm{pc}}\right\rangle\right)$ to $\left\langle b_{40 \mathrm{pc}}\right\rangle$. Here the individual regions show a stronger positive correlation between $\epsilon_{\mathrm{ff}}\left(\left\langle\Sigma_{40 \mathrm{pc}}\right\rangle\right)$ and $\left\langle b_{40 \mathrm{pc}}\right\rangle$. There is some indication that at least the interarm regions match the sense of the Padoan et al. (2012) prediction (the black line). The picture for the arm and center regions is less clear. Together they may show a weak positive correlation between $\epsilon_{\mathrm{ff}}\left(\left\langle\Sigma_{40 \mathrm{pc}}\right\rangle\right)$ and $\left\langle b_{40 \mathrm{pc}}\right\rangle$, but it is not clear that they should be grouped together. The offset between the interarm and arm regions at fixed $\left\langle b_{40 \mathrm{pc}}\right\rangle$ appears even stronger in $\epsilon_{\mathrm{ff}}\left(\left\langle\Sigma_{40 \mathrm{pc}}\right\rangle\right)$ than for $\tau_{\text {Dep }}^{\mathrm{mol}}$. At fixed $\left\langle b_{40 \mathrm{pc}}\right\rangle$ interarm regions have typically $\sim 0.24 \mathrm{dex}$, almost a factor of two, higher $\epsilon_{\mathrm{ff}}\left(\left\langle\Sigma_{40 \mathrm{pc}}\right\rangle\right)$ than arm regions with the same $\left\langle b_{40 \mathrm{pc}}\right\rangle$.

Together, Figures 8 and 9 paint a picture of M51 that qualitatively resembles that seen in many barred galaxies: despite the high surface densities in the inner dynamical features (here the arms), gas in this region appears stabilized against collapse. But flows along the arms feed gas condensations (the star-forming ring) in the inner regions (see Querejeta et al. 2016), where star-formation activity does proceed at a high level in both an absolute and normalized sense. Despite our averaging over moderately large $(370 \mathrm{pc})$ areas, timescale

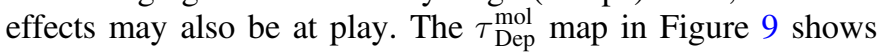
significant azimuthal structure, and as shown by Schinnerer et al. (2017) star formation tends to occur in spur-like structures downstream of the arms. We refer the reader to extensive discussions in Meidt et al. (2013), Colombo et al. (2014a), and Querejeta et al. (2016), Schinnerer et al. (2017), and references therein, for more discussion.

\section{Discussion and Summary}

We have used the PAWS survey (Schinnerer et al. 2013) to compare cloud-scale ISM structure to the locally averaged ability of gas to form stars across the inner part of M51. We compare infrared emission, tracing molecular gas mass, to recent infrared emission, tracing the recent SFR, within each $10^{\prime \prime} \approx 370 \mathrm{pc}$ and $30^{\prime \prime} \approx 1.1 \mathrm{kpc}$ beam. Then, we use the method described by Leroy et al. (2016) to calculate the massweighted $40 \mathrm{pc}$ surface density $\left(\left\langle\Sigma_{40 \mathrm{pc}}\right\rangle\right)$, line width $\left(\left\langle\sigma_{40 \mathrm{pc}}\right\rangle\right)$, and self-gravity $\left(\left\langle b_{40} \mathrm{pc}\right\rangle, b \equiv \Sigma / \sigma^{2} \propto \alpha_{\text {vir }}^{-1}\right)$ in each larger beam. This is similar to recording the mass-weighted mean GMC properties in each beam, but these intensity-based measurements are simpler and require fewer assumptions than estimating cloud properties. Still they capture the key physics in the Larson scaling relations well (Larson 1981).

We adopt simple translations between observed and physical quantities, so that our key results can be easily phrased in either observable or physical terms. Comparing CO and IR at large scales, we find the following.

1. At large scales, our $\mathrm{CO}$ and IR measurements qualitatively match previous studies of SFR-gas scaling relations in M51. The sublinear behavior noted by Shetty et al. (2013) at large radii, the superlinear behavior noted by Liu et al. (2011) in the inner galaxy, and the wide range of depletion times at high gas surface density found by Meidt et al. (2013) are all evident in Figure 2.

At 370 pc resolution, we still observe appreciable $(\sim 0.3$ dex $)$ scatter in the CO-to-IR ratio, rising $\sim 0.4$ dex at the highest surface densities. We compare the measured CO-to-IR ratio expressed as a molecular gas depletion time, $\tau_{\text {Dep }}^{\text {mol }}$, to the smallscale gas structure measured from PAWS to investigate if and how local gas structure drives depletion time variations. The most basic expectation, e.g., following Krumholz et al. (2012), is that variations in $\tau_{\text {Dep }}^{\text {mol }}$ result from variations in the cloudscale density, which sets the local gravitational free-fall time, $\tau_{\mathrm{ff}}$. To test this, we compare $\tau_{\text {Dep }}^{\text {mol }}$ to $\left\langle\Sigma_{40 \text { pc }}\right\rangle$, the mean cloud- 
scale surface density in the beam and our best observational tracer of the gas density. We find the following.

2. The CO-to-IR ratio, tracing $\tau_{\text {Dep }}^{\text {mol }}$, shows a weak anticorrelation with $\left\langle\Sigma_{40 \mathrm{pc}}\right\rangle$ over the range $\left\langle\Sigma_{40 \mathrm{pc}}\right\rangle \approx$ $100-350 M_{\odot} \mathrm{pc}^{-2}$ (Figure 3). Over this range, denser gas does appear moderately better at forming stars. The slope of this anticorrelation, approximately -0.25 to -0.35 , is shallower than what is naively expected for a fixed efficiency per free-fall time.

With an estimate of the line-of-sight depth, $h$, our $\left\langle\Sigma_{40 \mathrm{pc}}\right\rangle$ can be translated to a density, $\rho\left(\left\langle\Sigma_{40 \mathrm{pc}}\right\rangle\right)$, and then to a gravitational free-fall time, $\tau_{\text {ff }}$. Contrasting $\tau_{\text {Dep }}^{\text {mol }}$ and $\tau_{\text {ff }}$ yields an estimate of the efficiency of star formation per free-fall time, a central quantity for many recent theories of star formation. We consider what line-of-sight depth to use based on both recent GMC catalogs and studies of the disk thickness in M51 and the Milky Way.

3. In recent GMC catalogs targeting the Milky Way (Heyer et al. 2009; Miville-Deschênes et al. 2017) and M51 (Colombo et al. 2014a), the volume density and surface density of clouds correlate well (Figure 5). In these catalogs, most of the $\mathrm{CO}$ emission arises from clouds with $R \sim 30-100 \mathrm{pc}$. The observable cloud-scale surface density does appear to be a reasonable proxy for the local mean volume density, though more work is needed on this topic.

We adopt both a fiducial depth $h=100 \mathrm{pc}$ (our best estimate) and a "dynamical" depth calculated from holding the virial parameter constant. For both cases, we calculate the distribution of efficiency per free-fall time, $\epsilon_{\mathrm{ff}}\left(\left\langle\Sigma_{40 \mathrm{pc}}\right\rangle\right)$, across the PAWS field.

4. At both of our working resolutions, $\left\langle\epsilon_{\mathrm{ff}, 40 \mathrm{pc}}\right\rangle$ estimated in this way is $\sim 0.3 \%-0.36 \%$, with $\sim 0.3$ dex scatter for a $370 \mathrm{pc}$ averaging beam, and $\sim 0.1$ dex scatter for a $1.1 \mathrm{kpc}$ averaging beam (Figure 4).

This value agrees in broad terms with what one would infer based on comparing average GMC properties in the Milky Way and nearby galaxies (e.g., Bolatto et al. 2008; Heyer et al. 2009) to large-scale measurements of the molecular gas depletion time (e.g., Leroy et al. 2013). It also matches the apparent requirements for turbulent models to match observations of dense gas, IR, and $\mathrm{CO}$ in nearby galaxies (GarcíaBurillo et al. 2012; Usero et al. 2015). However, our inferred $\epsilon_{\mathrm{ff}}$ is much lower than values measured for the nearest molecular clouds by Evans et al. (2014), Murray (2011), or Lee et al. (2016; see also Lada et al. 2010, 2012), as well as for molecular clouds orbiting the Galactic Center by Barnes et al. (2017). It is also much lower than the values commonly adopted in analytic theories and numerical simulations (e.g., see Krumholz et al. 2012; Agertz \& Kravtsov 2015, among many others).

The main drivers for the mismatch with Lee et al. (2016) and Murray (2011) appear to be sampling effects. Our method averages over all evolutionary states to calculate a regional mean $\tau_{\text {Dep }}^{\text {mol }}$, while their work focuses on GMCs associated with peaks of recent star formation. The discrepancy with local clouds appears more subtle, but may be an issue of matching scales; the Evans et al. (2014) measurements focus on the $A_{V}>2$ mag material in local clouds, perhaps leading to the

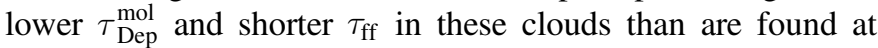

larger scales. The best ways to address these discrepancies appear to be high-resolution extinction-robust estimates of the SFR, to allow experiments exactly matched to those of Murray (2011) and Lee et al. (2016), and high-resolution (approximately a few parsec resolution) CO imaging of a large area (approximately kiloparsec) in a nearby galaxy, to investigate the superstructure around analogs to the Evans et al. (2014) clouds.

Beyond only the value of $\epsilon_{\mathrm{ff}}\left(\left\langle\Sigma_{40} \mathrm{pc}\right\rangle\right)$, we investigate how $\tau_{\text {Dep }}^{\text {mol }}$ and $\epsilon_{\mathrm{ff}}\left(\left\langle\Sigma_{40 \mathrm{pc}}\right\rangle\right)$ depend on the local cloud population and location in the galaxy. For $\tau_{\text {Dep }}^{\text {mol }}$, we find the following.

5. At high $\left\langle\Sigma_{40 \mathrm{pc}}\right\rangle>350 M_{\odot} \mathrm{pc}^{-2}$, the $\tau_{\text {Dep }}^{\text {mol }}$ increases with increasing $\left\langle\Sigma_{40 \text { pc }}\right\rangle$. This leads to the unexpected result, pointed out by Meidt et al. (2013), that some of the highest surface density regions of M51 show relatively weak star formation. These regions lie in the spiral arms and also have high $\left\langle\sigma_{40}\right.$ pc $\rangle$. Their low $\tau_{\text {Dep }}^{\text {mol }}$ is explained, in our analysis, by the fact that this gas appears more weakly self-gravitating (lower $\left\langle b_{40}\right.$ pc $\rangle$ ) than other material in M51 (Figures 7 and 8).

6. Instead of either surface density or line width alone, $\tau_{\text {Dep }}^{\text {mol }}$ appears most closely related to the ratio $b \equiv \Sigma / \sigma^{2}$ (Figure 7). Within a length scale (the line-of-sight depth through the disk), $\left\langle b_{40 \mathrm{pc}}\right\rangle$ traces the strength of selfgravity, $b \propto \alpha_{\text {vir }}^{-1} \propto \mathrm{UE} / \mathrm{KE} \propto \tau_{\text {ff }}^{2} / \tau_{\text {cross. }}^{2}$. Thus, gas that appears more gravitationally bound also appears better at forming stars. The power-law slope relating $\tau_{\text {Dep }}^{\text {mol }}$ to $\left\langle b_{40 \text { pc }}\right\rangle$ is $\tau_{\text {Dep }}^{\text {mol }} \propto b^{\beta}$ with $\beta=-0.8$ to -1.0 .

7. All three regions of the galaxy (arm, interarm, and center) line up in $\tau_{\text {Dep }}^{\text {mol }}-\left\langle b_{40}\right.$ pc $\rangle$ space, with only a modest, 0.13 dex ( $35 \%)$ offset (Figure 8$)$. This offset has the sense that the arm region has a modestly higher $\tau_{\text {Dep }}^{\text {mol }}$ (CO-to-IR) than the other regions at fixed $\left\langle b_{40} \mathrm{pc}\right\rangle$.

Our difference in results comparing $\tau_{\text {Dep }}^{\text {mol }}$ to $\left\langle b_{40 \text { pc }}\right\rangle$ and $\left\langle\Sigma_{40}\right.$ pc $\rangle$ suggest that $b$ does indeed trace the dynamical state. If both traced density, and if density represented the only important variable, then we would require $\tau_{\text {Dep }}^{\text {mol }}$ to depend on $\left\langle b_{40 \text { pc }}\right\rangle$ and $\left\langle\Sigma_{40 \text { pc }}\right\rangle$ in the same way. Instead, $\tau_{\text {Dep }}^{\text {mol }}$ show a steeper, more significant relation to $\left\langle b_{40 \mathrm{pc}}\right\rangle$ than to $\left\langle\Sigma_{40 \mathrm{pc}}\right\rangle$.

This apparent dependence of star formation on the dynamical state of the gas, or equivalently the virial parameter, echos findings for the Milky Way. There, the largest reservoir of high-density gas in the Galaxy is also currently the least efficient at forming stars. This phenomenon is thought to be caused by shear and the supervirial nature of the clouds (Kruijssen et al. 2014). Similarly, though our formalisms differ, our findings qualitatively agree with Meidt et al. (2013), who argued that the dynamical state of the gas in M51's arms, as observed by PAWS, suppresses star formation. Our results also agree with theoretical expectations in broad brush (e.g., Padoan et al. 2012; Krumholz \& McKee 2005; Federrath \& Klessen 2012). In detail, however, those models often make predictions about the efficiency of star formation per gravitational free-fall time. We compare $\epsilon_{\mathrm{ff}}\left(\left\langle\Sigma_{40 \mathrm{pc}}\right\rangle\right)$ to the local cloud populations and find the following.

9. In general $\epsilon_{\mathrm{ff}}\left(\left\langle\Sigma_{40 \mathrm{pc}}\right\rangle\right)$ appears anti-correlated with $\left\langle\Sigma_{40 \mathrm{pc}}\right\rangle$ and $\left\langle\sigma_{40 \mathrm{pc}}\right\rangle$ (Figure 7). The anticorrelation with $\left\langle\Sigma_{40 \mathrm{pc}}\right\rangle$ is weak over the range $\left\langle\Sigma_{40 \mathrm{pc}}\right\rangle \approx 100-350 M_{\odot}$ 
$\mathrm{pc}^{-2}$, but becomes stronger at high $\left\langle\Sigma_{40 \mathrm{pc}}\right\rangle$. The anticorrelation between $\epsilon_{\mathrm{ff}}\left(\left\langle\Sigma_{40 \mathrm{pc}}\right\rangle\right)$ and $\left\langle\sigma_{40 \mathrm{pc}}\right\rangle$ appears strong across the full range of $\left\langle\sigma_{40} \mathrm{pc}\right\rangle$ and becomes stronger at high dispersions. In general, a higher surface density and a higher line width both appear to imply lower efficiency per free-fall time in M51 (Figures 6 and 7).

10. We find a weak positive correlation between $\epsilon_{\mathrm{ff}}\left(\left\langle\Sigma_{40 \mathrm{pc}}\right\rangle\right)$ and $\left\langle b_{40} \mathrm{pc}\right\rangle$ for fixed line-of-sight depth, so that gas with higher apparent self-gravity appears to have a higher efficiency per free-fall time. Considering the whole galaxy, the strength of this correlation is weaker than the dependence predicted by the turbulent star-formation law of Padoan et al. (2012; Figure 7).

11. $\epsilon_{\mathrm{ff}}\left(\left\langle\Sigma_{40 \mathrm{pc}}\right\rangle\right)$ appears to correlate better with $b \propto \alpha_{\mathrm{vir}}^{-1}$ within an individual dynamical region, particularly within the interarm region (Figure 8). At fixed $\left\langle b_{40 \mathrm{pc}}\right\rangle$, we find $\epsilon_{\mathrm{ff}}\left(\left\langle\Sigma_{40 \mathrm{pc}}\right\rangle\right)$ to be $\sim 0.24$ dex lower in the arm regions than the interarm regions, on average. Thus relative to the expected collapse time, star formation is suppressed in the arms relative to the interarms by almost a factor of two at a fixed virial parameter (Figure 8).

Turbulent star-formation models tend to predict a positive correlation between $\epsilon_{\mathrm{ff}}$ and the Mach number, related to our observed line width. They also tend to predict a strong dependence of $\epsilon_{\mathrm{ff}}$ on $b \propto \alpha_{\mathrm{vir}}^{-1}$. Several theories have invoked an approximately fixed $\epsilon_{\mathrm{ff}}\left(\left\langle\Sigma_{40 \mathrm{pc}}\right\rangle\right)$. Thus, in detail, our observations do not show outstanding agreement with current models. However, those models include a number of additional dependencies, including on factors such as the magnetic field, character of the turbulence (see the summary in Federrath \& Klessen 2012). Our measurements also represent population, and so time, averages by design. So any dynamical cloud lifetime will be averaged out and lost (Murray 2011; Lee et al. 2016).

To facilitate a comparison with such models, we include all of our measurements in Table 1. We emphasize that our intensity-based approach is easy to replicate with no need for cloud-finding or other complex image processing. Indeed, numerical simulations can directly match our line-of-sight approach and thus marginalize over some of the geometrical uncertainties. Our approach to physical parameter estimation is simple and straightforward to treat via forward modeling. A main goal of this paper is to provide these measurements as an extragalactic benchmark for theories of star formation that consider cloud-scale gas structure.

Finally, as discussed in the text and the Appendix, there are systematic uncertainties regarding the CO-to- $\mathrm{H}_{2}$ conversion factor, SFR, and line-of-sight geometry. We motivate our choices in the text and the Appendix and test the impact of our assumptions, but these issues are standard in this field and should be born in mind when considering the results of the paper. We also anticipate refining technical details of our weighting averaging methods over the next year to better treating ensembles of line profiles and de-emphasize the impact of an extended averaging beam (see Leroy et al. 2016).

\subsection{Next Steps}

Within the next year, it should be possible to conduct a similar analysis as we present here for M51 for a diverse sample of local galaxies. These include the other five galaxies treated by Leroy et al. (2016) and targets of new ALMA mapping surveys that achieve cloud-scale resolution across $\sim 10$ star-forming galaxies. Such tests will establish: (1) if our observed very low $\epsilon_{\mathrm{ff}}\left(\left\langle\Sigma_{40 \mathrm{pc}}\right\rangle\right)$ is universal, (2) if the apparent role of self-gravity traced by $b$ is unique to M51 or a general feature, and (3) whether the gravitational free-fall time estimated from high-resolution imaging indeed appears to be a controlling parameter. The combination of these cloud-scale measurements with density-sensitive spectroscopy (e.g., Usero et al. 2015; Bigiel et al. 2016; Leroy et al. 2017) will also help connect structural analysis at the GMC-scale to the internal density structure of clouds, which plays a key role in their ability to form stars.

Our structural analysis follows the "beamwise" approach described in Leroy et al. (2016), but more literature exists estimating GMC properties for nearby galaxies (e.g., Fukui \& Kawamura 2010; Colombo et al. 2014a, the latter for M51). Following similar studies in the Milky Way (e.g., Murray 2011; Evans et al. 2014), these measurements can be compared to $\tau_{\text {Dep }}^{\text {mol }}$ in a similar way to what we do here. A. Schruba et al. (2017, in preparation) present such an analysis for a large collection of galaxies with GMC property measurements.

Finally, two major observational steps could address the tension between our measurements and those of the Milky Way. First, by observing CO from a large part of a star-forming galaxy at very high spatial resolution, one could attempt to mimic the Milky Way observations with full knowledge of the surrounding medium. Second, pairing extinction-robust SFR tracers with high-resolution gas mapping would allow for the kind of population studies carried out by Lee et al. (2016). The need to leverage low-resolution IR maps to estimate the SFR limits current efforts to consider population averages at a few hundred parsec scales.

We thank the anonymous referee for a thoughtful and constructive report. This work is based on observations carried out with the IRAM NOEMA Interferometer and the IRAM $30 \mathrm{~m}$ telescope. IRAM is supported by INSU/CNRS (France), MPG (Germany), and IGN (Spain). This work was carried out as part of the PHANGS collaboration (formerly SFNG) and the PAWS collaboration. The work of A.K.L., M.G., and J.S. is partially supported by the National Science Foundation under Grant Nos. 1615105, 1615109, and 1653300. E.S. acknowledge financial support to the DAGAL network from the People Programme (Marie Curie Actions) of the European Union's Seventh Framework Programme FP7/2007-2013/ under REA grant agreement number PITN-GA-2011-289313. E.S. acknowledges funding from the European Research Council (ERC) under the European Unions Horizon 2020 research and innovation programme (grant agreement No. 694343). A.H. acknowledges support from the Centre National d'Etudes Spatiales (CNES). J. M.D.K. and M.C. gratefully acknowledge financial support in the form of an Emmy Noether Research Group from the Deutsche Forschungsgemeinschaft (DFG), grant number KR4801/1-1 (PI: Kruijssen). J.M.D.K. acknowledges funding from the European Research Council (ERC) under the European Union's Horizon 2020 research and innovation programme via the ERC Starting Grant MUSTANG (grant agreement number 714907, PI Kruijssen). G.B. is supported by CONICYT/FONDECYT, Programa de Iniciacion, Folio 11150220. A.U. acknowledges support from Spanish MINECO grants AYA2012-32295 and FIS2012-32096. F.B. ac- knowledges support from DFG grant 

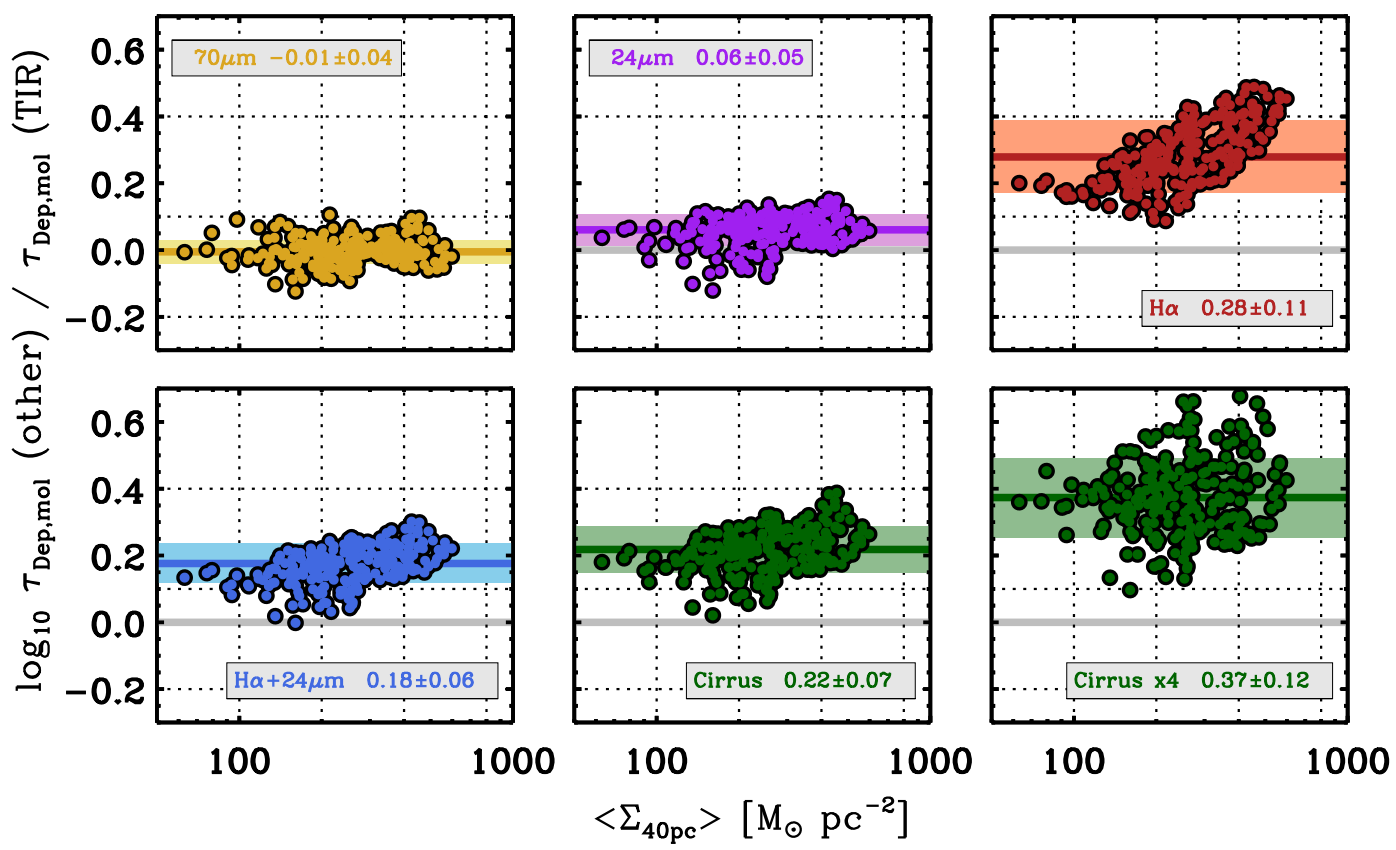

Figure 10. Effect of different SFR tracers on molecular gas depletion time. At $\theta=30^{\prime \prime}$ resolution, we estimate $\Sigma_{\text {SFR }}$ using: (top left) $70 \mu \mathrm{m}$ emission only, (top middle) $24 \mu \mathrm{m}$ emission only, (top right) $\mathrm{H} \alpha$ emission with one magnitude of extinction, and (bottom row) $\mathrm{H} \alpha+24 \mu \mathrm{m}$ with (left) no cirrus treatment, and (middle and right) 1 and 4 times a gas-based cirrus estimate removed from the $24 \mu \mathrm{m}$ emission. Prescriptions follow Leroy et al. (2012), and are similar to those from Murphy et al. (2011) and Calzetti et al. (2007). Each panel shows the ratio of $\tau_{\text {Dep }}^{\text {mol }}$ measured using the other SFR tracer to what we measure based on TIR emission at $30^{\prime \prime}$ resolution. The solid line and the shaded region show the median ratio and $\pm 1 \sigma$ range. Tracers involving $24 \mu \mathrm{m}$ tend to agree well with our estimates. Using $\mathrm{H} \alpha$ alone misses a substantial amount of extinction in regions of high gas surface density. Overall, most other estimates tend to modestly increase $\tau_{\text {Dep }}^{\text {mol }}$, which would imply lower $\epsilon_{\mathrm{ff}}\left(\left\langle\Sigma_{40 \mathrm{pc}}\right\rangle\right)$. None of the alternative SFR tracers appear to induce a downward tilt in the diagram, which we would expect for a fixed $\epsilon_{\mathrm{ff}}\left(\left\langle\Sigma_{40 \mathrm{pc}}\right\rangle\right)$.

BI1546/1-1. S.G.B. thanks economic support from grants ESP2015-68964-P and AYA2016-76682-C3-2-P.

\section{Appendix}

Our results depend on estimates of the recent SFR and molecular gas mass. We adopt simple approaches to each, utilizing the TIR emission as a tracer of the SFR and adopting a Galactic $\alpha_{\mathrm{CO}}=4.35 M_{\odot} \mathrm{pc}^{-2}\left(\mathrm{~K} \mathrm{~km} \mathrm{~s}^{-1}\right)^{-1}$ to convert $\mathrm{CO}(1$ $-0)$ intensity in molecular gas mass surface density.

\section{A.1. Other Approaches to the SFR}

We use TIR intensity as our tracer of SFR. At $\theta=30^{\prime \prime}$, we calculate $\Sigma_{\text {TIR }}$ using four bands and the SED-fitting based prescription of Galametz et al. (2013). At $\theta=10^{\prime \prime}$, we use a linear translation of $I_{70}$ into $\Sigma_{\mathrm{TIR}}$, with the coefficient derived from comparing $70 \mu \mathrm{m}$ intensity to TIR intensity at $30^{\prime \prime}$ resolution. We then translate $\Sigma_{\mathrm{TIR}}$ to $\Sigma_{\mathrm{SFR}}$, following Murphy et al. (2011).

The main impact of $\Sigma_{\mathrm{SFR}}$ in this paper is on the estimate of $\tau_{\text {Dep }}^{\text {mol }}$. To assess the impact of our choice of estimator, Figure 10 shows the effect on $\tau_{\text {Dep }}^{\text {mol }}$ of replacing our adopted TIR-based SFR with estimates using a different approach. We only have access to all of the required data at $\theta=30^{\prime \prime}$, so this plot shows only results for that resolution over the PAWS field, our area of interest.

First, we show results using only $70 \mu \mathrm{m}$ emission and the formulae quoted in Sections 2.2 and 2.3. This is our approach at $\theta=10^{\prime \prime}$, where Herschel's $70 \mu \mathrm{m}$ map is our only available IR band. We also show results using only $24 \mu \mathrm{m}$, using $\mathrm{H} \alpha$ assuming one magnitude of extinction, hybridizing $\mathrm{H} \alpha$ and $24 \mu \mathrm{m}$ emission, and combining $\mathrm{H} \alpha$ with $24 \mu \mathrm{m}$ after subtracting a "cirrus" (non-star-forming) component from the $24 \mu \mathrm{m}$ emission. Except for the $70 \mu \mathrm{m}$ emission, the prescriptions used for the other tracers are taken from Leroy et al. (2012), which builds heavily on Calzetti et al. (2007) and Murphy et al. (2011). We use the gas-based cirrus prediction, which assumes a typical dust-to-gas ratio and that all of the gas is illuminated by a radiation field 0.6 times that found in the Solar Neighborhood. The final panel shows the result for quadrupling the radiation field used in the cirrus estimate. Including an FUV-based hybrid (as in Leroy et al. 2008) would not add much to the analysis given the heavily extinguished nature of the region in question (see Leroy et al. 2012). Each panel quotes the median and scatter in the logarithm of the ratio between $\tau_{\text {Dep }}^{\text {mol }}$ estimated using this other tracer to that used in the main body of the paper.

The figure shows that the IR-based estimates agree well with one another and yield higher $\Sigma_{\mathrm{SFR}}$ than estimates using $\mathrm{H} \alpha$. Indeed, the main result of changing the SFR tracer is usually to lower $\Sigma_{\mathrm{SFR}}$, thereby increasing $\tau_{\mathrm{Dep}}^{\mathrm{mol}}$. The magnitude of the shift is a factor of $\sim 2$ if only $\mathrm{H} \alpha$ with 1 mag of extinction is used or a very large cirrus component is adopted (which also amounts to only weakly correcting $\mathrm{H} \alpha$ for extinction). A main result of our analysis is a low $\epsilon_{\mathrm{ff}}\left(\left\langle\Sigma_{40 \mathrm{pc}}\right\rangle\right)$. Lower $\Sigma_{\mathrm{SFR}}$ and higher $\tau_{\text {Dep }}^{\text {mol }}$ would drive $\epsilon_{\mathrm{ff}}\left(\left\langle\Sigma_{40 \mathrm{pc}}\right\rangle\right)$ to even lower values. In detail, given the gas-rich, dusty nature of the inner few kiloparsecs of M51, we do not necessarily expect these lower $\Sigma_{\mathrm{SFR}}$ estimates to be more correct, but if they are then it would not change our qualitative conclusions.

Note that data at higher $\left\langle\Sigma_{40 \mathrm{pc}}\right\rangle$ tend to show a larger discrepancy between IR-based SFR estimates and $\mathrm{H} \alpha$ with little or no correction. The sense of this trend is that most alternatives to the IR-based $\Sigma_{\mathrm{SFR}}$ would yield longer $\tau_{\text {Dep }}^{\text {mol }}$ at 

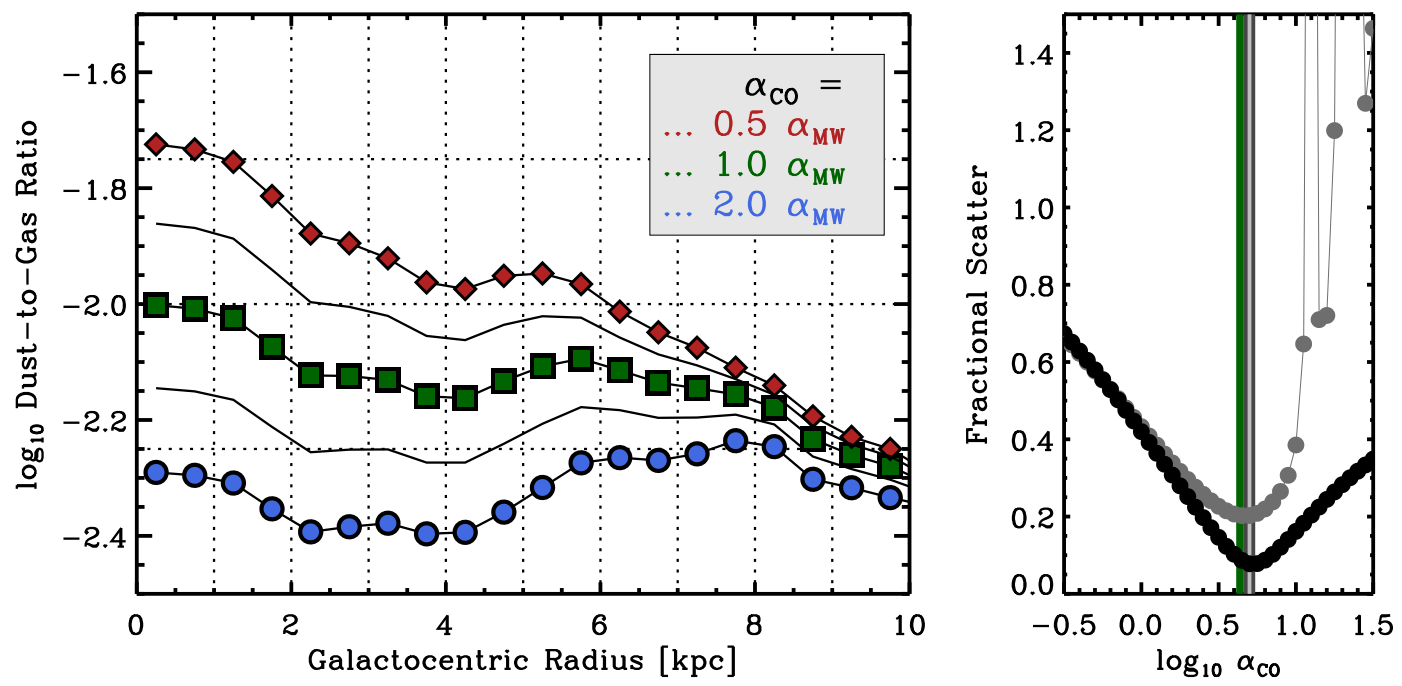

Figure 11. Constraints on $\alpha_{\mathrm{CO}}$ from comparing CO, HI, and dust mass surface density from fitting the Mentuch Cooper et al. (2012) Herschel data using modified versions of the Draine \& $\mathrm{Li}$ (2007) models. (Left) The dust-to-gas ratio as a function of galactocentric radius for different values of $\alpha_{\mathrm{CO}}$ : from top to bottom the 0.5 (red), $\sqrt{2}^{-1}, 1$ (green), $\sqrt{2}$, and 2 (blue) times the Milky Way $\alpha_{\mathrm{CO}}=4.35 M_{\odot} \mathrm{pc}^{-2}\left(\mathrm{~K} \mathrm{~km} \mathrm{~s}^{-1}\right)^{-1}$. Our adopted Milky Way $\alpha_{\mathrm{CO}}$ yields a nearly flat dust-to-gas ratio, consistent with the weak metallicity gradient in the galaxy Croxall et al. (2015). (Right) Solution for $\alpha_{\mathrm{CO}}$ (in units of $\left.M_{\odot} \mathrm{pc}^{-2}\left(\mathrm{~K} \mathrm{~km} \mathrm{~s}^{-1}\right)^{-1}\right)$ for individual pixels (gray) and radial profile bins (black) assuming a fixed dust-to-gas ratio. We use the fractional minimization technique of Sandstrom et al. (2013) and find the least scatter in the dust-to-gas ratio for $\alpha_{\mathrm{CO}}=4.5-5 \mathrm{M}_{\odot} \mathrm{pc}^{-2}\left(\mathrm{~K} \mathrm{~km} \mathrm{~s}^{-1}\right)^{-1}$. For a more detailed analysis using multiple techniques, we refer the reader to B. Groves et al. (2017, in preparation).

higher $\left\langle\Sigma_{40 \text { pc }}\right\rangle$. The result would be an even lower $\epsilon_{\mathrm{ff}}\left(\left\langle\Sigma_{40 \mathrm{pc}}\right\rangle\right)$ at high $\left\langle\Sigma_{40 \text { pc }}\right\rangle$ than we already observe. That is, none of the alternatives in Figure 10 push the data toward a more nearly fixed $\epsilon_{\mathrm{ff}}\left(\left\langle\Sigma_{40 \mathrm{pc}}\right\rangle\right)$.

Furthermore, recall that Figure 2 shows that our IR-based approach yields measurements that overlap the $\mathrm{Pa} \alpha+24 \mu \mathrm{m}$ based estimates from Kennicutt et al. (2007). They studied selected apertures, while we sample the whole inner disk, so there are methodological differences. But the overall magnitude of both the gas and SFR estimates agrees well.

Finally, note from the first panel that $\tau_{\text {Dep }}^{\text {mol }}$ estimated using only $70 \mu \mathrm{m}$ emission and our adopted scaling agrees very well with that estimated using the four-band Galametz et al. (2013) fit. That is, the approach that we use at $\theta=30^{\prime \prime}$ agrees well with that which we are forced to use $\theta=10^{\prime \prime}$. The median ratio agrees by construction, but the small scatter gives us confidence in our use of $70 \mu \mathrm{m}$ emission and our application of Equation (1) (though see Boquien et al. 2016, for a more in depth consideration of IR emission as a function of scale).

\section{A.2. The CO-to- $\mathrm{H}_{2}$ Conversion Factor}

We translate $\mathrm{CO}(1-0)$ emission into molecular mass assuming a fixed $\alpha_{\mathrm{CO}}=4.35 M_{\odot} \mathrm{pc}^{-2}\left(\mathrm{~K} \mathrm{~km} \mathrm{~s}^{-1}\right)^{-1}$. This value is supported by multi-line (Schinnerer et al. 2010) and cloud virial mass (Colombo et al. 2014a) studies. Schinnerer et al. (2010) provide a thorough summary of the literature on $\alpha_{\mathrm{CO}}$ in M51, which has so far yielded results that break down into either an approximately Galactic conversion factor or values $\sim 0.5$ times Galactic. If the lower $\alpha_{\mathrm{CO}}$ holds, there would be less molecular gas mass than we infer in the main paper, and a shorter $\tau_{\text {Dep }}^{\text {mol }}$. This would increase $\epsilon_{\mathrm{ff}}\left(\left\langle\Sigma_{40 \mathrm{pc}}\right\rangle\right)$ by $\left(\alpha_{\mathrm{CO}} / \alpha_{\mathrm{MW}}\right)^{-1.5}$, because the conversion factor also affects the density and so $\tau_{\mathrm{ff}} \propto 1 / \sqrt{\rho}$.
Figure 11 shows that an approximately Galactic conversion factor is also supported by the dust-based approach of Sandstrom et al. (2013) and Leroy et al. (2011). We compare $\Sigma_{\text {dust }}$, the dust mass surface density estimated from Herschel multiband data, to the measured $\mathrm{CO}$ intensity and the $\mathrm{HI}$ column density from VLA imaging. The CO map is the PAWS single dish map, the HI map comes from THINGS (Walter et al. 2008). The dust map is the result of fitting using the Draine \& Li (2007), Draine et al. (2007) models to the Herschel and Spitzer photometry, following Aniano et al. (2012) and modified by the correction to dust mass suggested in Planck Collaboration et al. (2016).

For this application, we assume that the dust-to-gas ratio is constant over the range $r_{\mathrm{gal}}=1-8 \mathrm{kpc}$. The approximately constant metallicity of the galaxy supports this assumption (e.g., Croxall et al. 2015). The figure shows that $\alpha_{\mathrm{CO}} \approx \alpha_{\mathrm{MW}}=4.35 M_{\odot} \mathrm{pc}^{-2}\left(\mathrm{~K} \mathrm{~km} \mathrm{~s}^{-1}\right)^{-1}$ yields an approximately flat dust-to-gas ratio as a function of radius. A lower conversion factor, as suggested by Nakai \& Kuno (1995) and Wall et al. (2016) yields a strong gradient in dust-to-gas ratio as a function of radius. The right panel shows the formal results of minimizing scatter in DGR, while varying $\alpha_{\mathrm{CO}}$ treating either each ring (black) or each $\theta=30^{\prime \prime}$ line of sight (gray) as independent measurements. Both approaches yield a best fit $\alpha_{\mathrm{CO}} \approx 4.5-5.0 M_{\odot} \mathrm{pc}^{-2}\left(\mathrm{~K} \mathrm{~km} \mathrm{~s}^{-1}\right)^{-1}$.

Uncertainties apply to this dust-based approach, including phase- or density-dependent depletion (Jenkins 2009), emissivity variations (e.g., Ossenkopf \& Henning 1994), and the presence of sufficient dynamic range in the $\mathrm{H}_{2} / \mathrm{HI}$ ratio to achieve a good fit (Sandstrom et al. 2013). The interplay of these uncertainties with $\alpha_{\mathrm{CO}}$ variation are discussed at length in Israel (1997), Leroy et al. (2007, 2011), Sandstrom et al. (2013), and Roman-Duval et al. (2014), and are beyond the scope of this paper. The key point, for us, is that the best 
current available dust and gas maps suggest that our adopted $\alpha_{\mathrm{CO}} \approx \alpha_{\mathrm{MW}}$ represents a reasonable choice.

\section{ORCID iDs}

Adam K. Leroy (1) https://orcid.org/0000-0002-2545-1700 Eva Schinnerer (i) https://orcid.org/0000-0002-3933-7677 Annie Hughes (i) https://orcid.org/0000-0002-9181-1161 Sharon Meidt (i) https://orcid.org/0000-0002-6118-4048 Jiayi Sun 주 https://orcid.org/0000-0003-0378-4667 Frank Bigiel (1) https://orcid.org/0000-0003-0166-9745 Alberto Bolatto (10) https://orcid.org/0000-0002-5480-5686 Molly Gallagher (ib https://orcid.org/0000-0001-5285-5930 Santiago Garcia-Burillo (1) https://orcid.org/0000-00030444-6897

Jerome Pety (1) https://orcid.org/0000-0003-3061-6546 Antonio Usero (10) https://orcid.org/0000-0003-1242-505X

\section{References}

Agertz, O., \& Kravtsov, A. V. 2015, ApJ, 804, 18

Aniano, G., Draine, B. T., Calzetti, D., et al. 2012, ApJ, 756, 138

Aniano, G., Draine, B. T., Gordon, K. D., \& Sandstrom, K. 2011, PASP, 123,1218

Barnes, A. T., Longmore, S. N., Battersby, C., et al. 2017, MNRAS, 469, 2263 Bigiel, F., Leroy, A., Walter, F., et al. 2008, AJ, 136, 2846

Bigiel, F., Leroy, A. K., Jiménez-Donaire, M. J., et al. 2016, ApJL, 822, L26

Blanc, G. A., Heiderman, A., Gebhardt, K., Evans, N. J., \& Adams, J. 2009, ApJ, 704, 842

Bolatto, A. D., Leroy, A. K., Rosolowsky, E., Walter, F., \& Blitz, L. 2008, ApJ, 686, 948

Bolatto, A. D., Warren, S. R., Leroy, A. K., et al. 2013b, Natur, 499, 450

Bolatto, A. D., Wolfire, M., \& Leroy, A. K. 2013a, ARA\&A, 51, 207

Boquien, M., Kennicutt, R., Calzetti, D., et al. 2016, A\&A, 591, A6

Bothwell, M. S., Wagg, J., Cicone, C., et al. 2014, MNRAS, 445, 2599

Brunt, C. M. 2003, ApJ, 584, 293

Brunt, C. M., Heyer, M. H., \& Mac Low, M.-M. 2009, A\&A, 504, 883

Calzetti, D., Kennicutt, R. C., Engelbracht, C. W., et al. 2007, ApJ, 666, 870

Calzetti, D., Kennicutt, R. C., Jr., Bianchi, L., et al. 2005, ApJ, 633, 871

Ciardullo, R., Feldmeier, J. J., Jacoby, G. H., et al. 2002, ApJ, 577, 31

Colombo, D., Hughes, A., Schinnerer, E., et al. 2014a, ApJ, 784, 3

Colombo, D., Meidt, S. E., Schinnerer, E., et al. 2014b, ApJ, 784, 4

Croxall, K. V., Pogge, R. W., Berg, D. A., Skillman, E. D., \& Moustakas, J. 2015, ApJ, 808, 42

Davis, S. W., Jiang, Y.-F., Stone, J. M., \& Murray, N. 2014, ApJ, 796, 107

Draine, B. T., Dale, D. A., Bendo, G., et al. 2007, ApJ, 663, 866

Draine, B. T., \& Li, A. 2007, ApJ, 657, 810

Evans, N. J., II, Heiderman, A., \& Vutisalchavakul, N. 2014, ApJ, 782, 114

Faesi, C. M., Lada, C. J., Forbrich, J., Menten, K. M., \& Bouy, H. 2014, ApJ, 789,81

Federrath, C., \& Klessen, R. S. 2012, ApJ, 761, 156

Federrath, C., \& Klessen, R. S. 2013, ApJ, 763, 51

Feldmeier, J. J., Ciardullo, R., \& Jacoby, G. H. 1997, ApJ, 479, 231

Foyle, K., Rix, H.-W., Walter, F., \& Leroy, A. K. 2010, ApJ, 725, 534

Fukui, Y., \& Kawamura, A. 2010, ARA\&A, 48, 547

Galametz, M., Kennicutt, R. C., Calzetti, D., et al. 2013, MNRAS, 431, 1956

Gao, Y., \& Solomon, P. M. 2004, ApJ, 606, 271

García-Burillo, S., Usero, A., Alonso-Herrero, A., et al. 2012, A\&A, 539, A8

Gardan, E., Braine, J., Schuster, K. F., Brouillet, N., \& Sievers, A. 2007, A\&A, 473, 91

Hagiwara, Y. 2007, AJ, 133, 1176

Heiderman, A., Evans, N. J., II, Allen, L. E., Huard, T., \& Heyer, M. 2010, ApJ, 723, 1019

Helfer, T. T., Thornley, M. D., Regan, M. W., et al. 2003, ApJS, 145, 259

Hennebelle, P., \& Chabrier, G. 2011, ApJL, 743, L29

Heyer, M., \& Dame, T. M. 2015, ARA\&A, 53, 583

Heyer, M., Krawczyk, C., Duval, J., \& Jackson, J. M. 2009, ApJ, 699, 1092

Hughes, A., Meidt, S. E., Colombo, D., et al. 2013a, ApJ, 779, 46
Hughes, A., Meidt, S. E., Schinnerer, E., et al. 2013b, ApJ, 779, 44 Hunt, L. K., García-Burillo, S., Casasola, V., et al. 2015, A\&A, 583, A114 Israel, F. P. 1997, A\&A, 328, 471

Jenkins, E. B. 2009, ApJ, 700, 1299

Kawamura, A., Mizuno, Y., Minamidani, T., et al. 2009, ApJS, 184, 1 Kennicutt, R. C., Jr. 1998, ARA\&A, 36, 189

Kennicutt, R. C., Jr., Armus, L., Bendo, G., et al. 2003, PASP, 115, 928

Kennicutt, R. C., Jr., Calzetti, D., Walter, F., et al. 2007, ApJ, 671, 333

Koda, J., Scoville, N., Sawada, T., et al. 2009, ApJL, 700, L132

Koyama, H., \& Ostriker, E. C. 2009, ApJ, 693, 1346

Kroupa, P. 2001, MNRAS, 322, 231

Kruijssen, J. M. D., \& Longmore, S. N. 2014, MNRAS, 439, 3239

Kruijssen, J. M. D., Longmore, S. N., Elmegreen, B. G., et al. 2014, MNRAS, 440, 3370

Krumholz, M. R., Dekel, A., \& McKee, C. F. 2012, ApJ, 745, 69

Krumholz, M. R., \& McKee, C. F. 2005, ApJ, 630, 250

Krumholz, M. R., \& Tan, J. C. 2007, ApJ, 654, 304

Lada, C. J., Forbrich, J., Lombardi, M., \& Alves, J. F. 2012, ApJ, 745, 190

Lada, C. J., Lombardi, M., \& Alves, J. F. 2010, ApJ, 724, 687

Larson, R. B. 1981, MNRAS, 194, 809

Lee, E. J., Miville-Deschênes, M.-A., \& Murray, N. W. 2016, ApJ, 833, 229

Leroy, A., Bolatto, A., Stanimirović, S., et al. 2007, ApJ, 658, 1027

Leroy, A. K., Bigiel, F., de Blok, W. J. G., et al. 2012, AJ, 144, 3

Leroy, A. K., Bolatto, A., Gordon, K., et al. 2011, ApJ, 737, 12

Leroy, A. K., Bolatto, A. D., Ostriker, E. C., et al. 2015, ApJ, 801, 25

Leroy, A. K., Hughes, A., Schruba, A., et al. 2016, ApJ, 831, 16

Leroy, A. K., Usero, A., Schruba, A., et al. 2017, ApJ, 835, 217

Leroy, A. K., Walter, F., Brinks, E., et al. 2008, AJ, 136, 2782

Leroy, A. K., Walter, F., Sandstrom, K., et al. 2013, AJ, 146, 19

Lewis, A. R., Simones, J. E., Johnson, B. D., et al. 2017, ApJ, 834, 70

Liu, G., Koda, J., Calzetti, D., Fukuhara, M., \& Momose, R. 2011, ApJ, 735, 63

Mac Low, M.-M., \& Klessen, R. S. 2004, RvMP, 76, 125

McKee, C. F., \& Ostriker, E. C. 2007, ARA\&A, 45, 565

Meidt, S., Leroy, A. K., Rosolowsky, E., et al. 2017, ApJ, submitted

Meidt, S. E., Hughes, A., Dobbs, C. L., et al. 2015, ApJ, 806, 72

Meidt, S. E., Schinnerer, E., García-Burillo, S., et al. 2013, ApJ, 779, 45

Mentuch Cooper, E., Wilson, C. D., Foyle, K., et al. 2012, ApJ, 755, 165

Miville-Deschênes, M.-A., Murray, N., \& Lee, E. J. 2017, ApJ, 834, 57

Momose, R., Koda, J., Kennicutt, R. C., Jr., et al. 2013, ApJL, 772, L13

Murphy, E. J., Condon, J. J., Schinnerer, E., et al. 2011, ApJ, 737, 67

Murray, N. 2011, ApJ, 729, 133

Nakai, N., \& Kuno, N. 1995, PASJ, 47, 761

Ossenkopf, V., \& Henning, T. 1994, A\&A, 291, 943

Ossenkopf, V., \& Mac Low, M.-M. 2002, A\&A, 390, 307

Padoan, P., \& Nordlund, A. 2002, ApJ, 576, 870

Padoan, P., \& Nordlund, Å. 2011, ApJ, 730, 40

Padoan, P., Haugbølle, T., \& Nordlund, A. 2012, ApJL, 759, L27

Pety, J., Schinnerer, E., Leroy, A. K., et al. 2013, ApJ, 779, 43

Planck Collaboration, Ade, P. A. R., Aghanim, N., et al. 2016, A\&A, 586, A132

Querejeta, M., Schinnerer, E., García-Burillo, S., et al. 2016, A\&A, 593, A118 Roman-Duval, J., Gordon, K. D., Meixner, M., et al. 2014, ApJ, 797, 86

Saintonge, A., Kauffmann, G., Wang, J., et al. 2011, MNRAS, 415, 61

Sandstrom, K. M., Leroy, A. K., Walter, F., et al. 2013, ApJ, 777, 5

Schinnerer, E., Meidt, S. E., Colombo, D., et al. 2017, ApJ, 836, 62

Schinnerer, E., Meidt, S. E., Pety, J., et al. 2013, ApJ, 779, 42

Schinnerer, E., Weiß, A., Aalto, S., \& Scoville, N. Z. 2010, ApJ, 719, 1588

Schruba, A., Leroy, A. K., Kruijssen, J. M. D., et al. 2017, ApJ, 835, 278

Schruba, A., Leroy, A. K., Walter, F., et al. 2011, AJ, 142, 37

Schruba, A., Leroy, A. K., Walter, F., et al. 2012, AJ, 143, 138

Schruba, A., Leroy, A. K., Walter, F., Sandstrom, K., \& Rosolowsky, E. 2010, ApJ, 722, 1699

Shetty, R., Kelly, B. C., \& Bigiel, F. 2013, MNRAS, 430, 288

Solomon, P. M., Rivolo, A. R., Barrett, J., \& Yahil, A. 1987, ApJ, 319, 730

Suwannajak, C., Tan, J. C., \& Leroy, A. K. 2014, ApJ, 787, 68

Usero, A., Leroy, A. K., Walter, F., et al. 2015, AJ, 150, 115

Wall, W. F., Puerari, I., Tilanus, R., et al. 2016, MNRAS, 459, 1440

Walter, F., Brinks, E., de Blok, W. J. G., et al. 2008, AJ, 136, 2563

Young, J. S., Allen, L., Kenney, J. D. P., Lesser, A., \& Rownd, B. 1996, AJ, 112,1903 